\title{
TOPOLOGY OF RIEMANNIAN SUBMANIFOLDS WITH PRESCRIBED BOUNDARY
}

\author{
STEPHANIE ALEXANDER, MOHAMMAD GHOMI, AND JEREMY WONG
}

\begin{abstract}
We prove that a smooth compact submanifold of codimension 2 immersed in $\mathbf{R}^{n}, n \geq 3$, bounds at most finitely many topologically distinct compact nonnegatively curved hypersurfaces. This settles a question of Guan and Spruck related to a problem of Yau. Analogous results for complete fillings of arbitrary Riemannian submanifolds are obtained as well. On the other hand, we show that these finiteness theorems may not hold if the codimension is too high, or the prescribed boundary is not sufficiently regular. Our proofs employ, among other methods, a relative version of Nash's isometric embedding theorem, and the theory of Alexandrov spaces with curvature bounded below, including the compactness and stability theorems of Gromov and Perelman.
\end{abstract}

\section{INTRODUCTION}

Recently there has been considerable interest in studying the structure of locally convex hypersurfaces with prescribed boundary in Euclidean space 11, 2, 14, 15, 17. 18, 20, 22, 26, 31, 35]. A fundamental problem in this area, posed by S.T. Yau [41, Problem 26], is deciding when a closed curve in $\mathbf{R}^{3}$, or more generally a submanifold $\Gamma$ of codimension 2 in $\mathbf{R}^{n}$, bounds a hypersurface of positive curvature. A related question, raised more recently by Guan and Spruck [20, p. 1312], is whether $\Gamma$ bounds at most only finitely many topological types of locally convex hypersurfaces. In this paper we show that the answer is yes provided that $\Gamma$ is sufficiently regular, and also investigate this problem for arbitrary Riemannian submanifolds of any codimension. Our first result illustrates the need for regularity:

Theorem 1.1. There exists a simple closed rectifiable curve in $\mathbf{R}^{3}$ that is differentiable in its arclength parameter, is $\mathcal{C}^{\infty}$ in the complement of two points, and bounds infinitely many topologically distinct, compact, embedded, positively curved $\mathcal{C}^{\infty}$ surfaces.

In contrast, the following theorem shows that if the regularity of the bounding curve is increased slightly so that it has finite turn, then there are only finitely many such "fillings" or "spanning surfaces", even if their regularity is relaxed to the locally

Date: Last Typeset October 16, 2009.

1991 Mathematics Subject Classification. Primary 53A07, 53C23; Secondary 53C21, 53C45.

Key words and phrases. Nonnegative curvature, locally convex hypersurface, filling Riemannian manifold, finiteness theorem, Alexandrov space with curvature bounded below.

The second named author was supported in part by NSF CAREER award DMS-0332333, and grant DMS-0806305. 
convex category. Finite turn corresponds to rectifiability of the tangent indicatrix, and is considerably weaker than, for example, piecewise $\mathcal{C}^{1,1}$. By a locally convex immersed hypersurface $M$ with boundary, we mean a map $f: M \rightarrow \mathbf{R}^{n+1}$ that has an extension to a continuous map $\widetilde{f}$ of a manifold without boundary $\widetilde{M}$, where every point of $M$ has a neighborhood in $\widetilde{M}$ that is embedded by $\widetilde{f}$ into the boundary of some convex body.

Theorem 1.2. A finite collection of closed curves of finite turn immersed in $\mathbf{R}^{3}$ bounds at most finitely many topologically distinct, compact, locally convex immersed surfaces.

Assuming greater regularity, one can prove a much stronger finiteness result for spanning surfaces. In the following theorem, the ambient space is an arbitrary Riemannian manifold, of arbitrary codimension, and the spanning surfaces need not be compact.

Theorem 1.3. A finite collection of closed $\mathcal{C}^{3}$ curves immersed in a given Riemannian manifold bounds at most finitely many topologically distinct, complete, immersed $\mathcal{C}^{3}$ surfaces whose total curvature is uniformly bounded below.

The proofs of Theorems 1.2 and 1.3 draw on the classical resource of GaussBonnet. To investigate to what extent Theorem 1.3 extends to higher dimensions, we turn to Gromov-Hausdorff convergence theory. We start with the following finiteness theorem for Riemannian fillings of arbitrary dimension and codimension in the presence of bounds on curvature and diameter:

Theorem 1.4. Let $\Gamma$ be a compact (but not necessarily connected) $\mathcal{C}^{3}$ submanifold of a given Riemannian manifold. Then $\Gamma$ bounds at most finitely many distinct topological types of compact immersed submanifolds having uniform bounds below on curvature and above on intrinsic diameter.

By curvature throughout this paper we mean sectional curvature unless stated otherwise. The diameter bound in Theorem 1.4 cannot in general be removed, even for positively curved fillings in Euclidean spaces, as the following example shows.

Theorem 1.5. The standard embedding of $\mathbf{S}^{2}$ in $\mathbf{R}^{3} \subset \mathbf{R}^{18}$ bounds infinitely many topologically distinct $\mathcal{C}^{\infty}$ compact 3 -dimensional submanifolds with positive curvature.

However, it is possible to remove the diameter bound in Theorem 1.4 when $\Gamma$ is a submanifold of codimension 2 in Euclidean space and the filling hypersurface is nonnegatively curved. In this setting, we exploit convexity as an additional resource in order to prove our next two theorems.

Theorem 1.6. A compact (but not necessarily connected), $\mathcal{C}^{3}$ immersed submanifold of codimension 2 in $\mathbf{R}^{n+1}, n>2$, bounds at most finitely many topologically distinct, compact, nonnegatively curved $\mathcal{C}^{3}$ immersed hypersurfaces.

Moreover, finiteness of topological types holds in the class of noncompact, nonnegatively curved fillings, provided the curvature at each point does not vanish on 
too many sections. The following theorem includes all strictly positively curved spanning hypersurfaces, but also includes hypersurfaces with no points of strictly positive curvature.

Theorem 1.7. A compact (but not necessarily connected), $\mathcal{C}^{3}$ immersed submanifold of codimension 2 in $\mathbf{R}^{n+1}, n \geq 2$, bounds at most finitely many topologically distinct, complete, nonnegatively curved $\mathcal{C}^{3}$ immersed hypersurfaces for which the second fundamental form has nullity at most 1 everywhere.

Finally, we provide further evidence that Euclidean submanifolds of codimension 2 are indeed the correct setting for the noncompact finiteness phenomenon of Theorem 1.7. by giving a counterexample to a closely related intrinsic finiteness statement about complete open Riemannian manifolds.

The proofs of these results are presented in the following sections. Theorem 1.1 is proved in Section 2 by means of a direct construction which develops certain gluing techniques, including a bridge principle, for positively curved surfaces with boundary. Theorems 1.2 and 1.3 , which are concerned with 2-dimensional fillings and draw on Gauss-Bonnet theory, are proved in Section 3 . Theorems 1.4 and 1.6 concern higher-dimensional compact fillings. They are proved in Section 4 with the aid of results of Gromov and Perelman on compactness and stability of Alexandrov spaces with curvature bounded below, together with extension results for manifolds with boundary and some diameter estimates. Theorem 1.5 is also proved in this section, using a relative version of the Nash isometric embedding theorem. Theorem 1.7. which concerns complete open fillings, is proved in Section 5 via a clipping procedure, and a theorem of Cai on finiteness of the number of ends, which are used to reduce the proof to one similar to that of the compact case considered in Section 4. Section 6 contains further comments and questions.

\section{The Regularity Example: Proof of Theorem 1.1}

In this section we construct a differentiable curve in $\mathbf{R}^{3}$ with infinitely many topologically distinct positively curved filling surfaces. The proof is presented in the setting of a surgery procedure for locally convex surfaces. In particular we use a gluing result (Theorem 2.1) which in turn is proved with the aid of a bridge principle (Proposition 2.7).

2.1. Overview. Figure 1 shows a picture of the curve $\Gamma$, together with three of its positively curved fillings, which we will construct here. Each of these filling surfaces is symmetric with respect to a horizontal plane. The first surface on the left, say $M_{0}$, is homeomorphic to a disk. It consists of two spherical pieces which are connected by a strip of positive curvature. The surface in the middle, $M_{1}$, is obtained by adding a handle to $M_{0}$ (without perturbing $\Gamma$ ) and is thus homeomorphic to a punctured torus. Similarly, the surface on the right, $M_{2}$, is obtained by adding a handle to $M_{1}$. This process of addition of handles, or more precisely surgery where we remove a pair of disks from a surface and glue an annulus in their place, may be continued indefinitely because $\Gamma$ has an infinite pair of corrugations where handles may be added. Thus we obtain an infinite sequence of surfaces $M_{i}$, with $\partial M_{i}=\Gamma$ such 

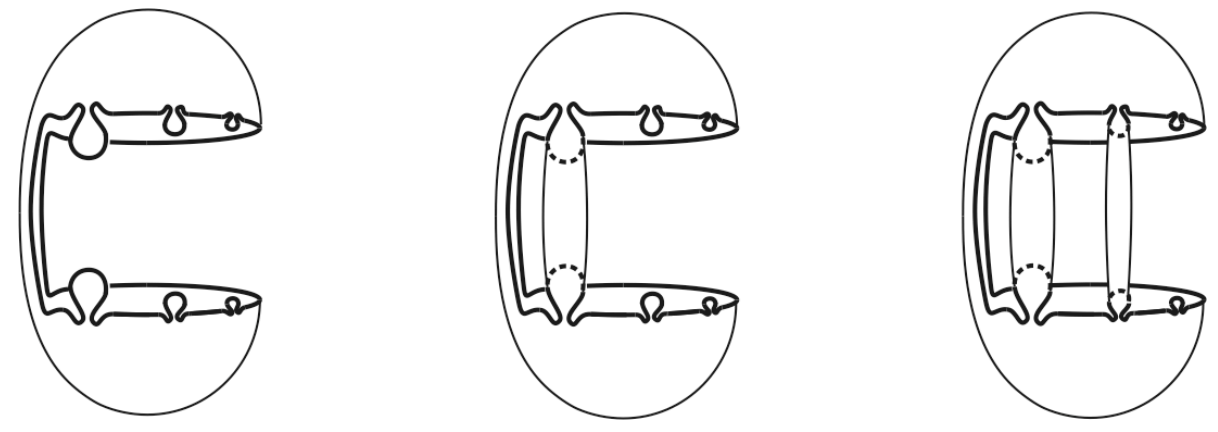

FIGURE 1.

that $g\left(M_{i}\right)=i$, where $g$ denotes the topological genus. Further we note that the corrugations of $\Gamma$ converge to a pair of points on $\Gamma$ with a sufficiently fast rate so that $\Gamma$ is rectifiable, is differentiable at the accumulation points with respect to the arclength parameter, and is $\mathcal{C}^{\infty}$ elsewhere. Furthermore, each of the surfaces $M_{i}$ is $\mathcal{C}^{\infty}$ and has positive curvature up to the boundary; this means that for every $p \in \Gamma$ there exists an open neighborhood $U \subset M_{i}$ containing $p$, and a $\mathcal{C}^{\infty}$-smooth positively curved surface $\widetilde{U}$ without boundary such that $\widetilde{U} \cap M_{i}=U$.

2.2. Construction of $\Gamma$. The general strategy here is to construct one half of the surface $M_{0}$ mentioned above, reflect it, and let $\Gamma$ be the boundary of the resulting surface. This is achieved in the following steps:

Step 1. Let $S \subset \mathbf{R}^{3}$ be a sphere whose center lies below the $x y$-plane, e.g. at $(0,0,-1)$. Let $p_{0} \in S$ be a point on the equator $\Gamma_{0}$ of $S$. Then for every $r>0$ we may find an ellipsoid of revolution, symmetric with respect to the $x y$-plane, and with a vertical axis of revolution, which is tangent to exactly one point of $S$, say $q_{0}$, and intersects $S$ in a figure-eight curve which is contained entirely in $B_{r}\left(p_{0}\right)$, a ball of radius $r$ centered at $p_{0}$. Let the half of this ellipsoid which lies below the $x y$-plane be denoted by $E_{0}$. See the picture on the left in Figure 2 .
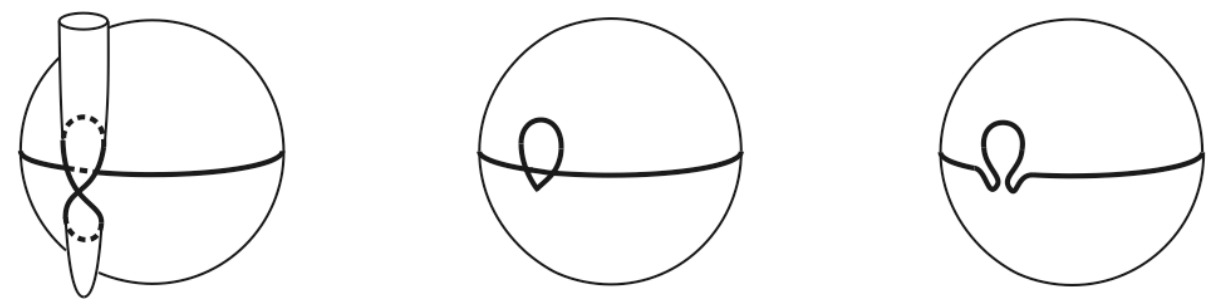

FigURE 2.

Step 2. We deform $E_{0}$ to obtain a smooth surface of positive curvature $\bar{E}_{0}$ such that (i) $\bar{E}_{0}$ coincides with $S$ in an open neighborhood $U$ of $q_{0}$, (ii) $\bar{E}_{0}$ coincides with $E_{0}$ outside of $B_{r}\left(p_{0}\right)$, and (iii) $E_{0} \cap S \subset \bar{E}_{0}$. This follows immediately from the following result, which will be proved later. 
Theorem 2.1. Let $\Sigma_{1}$ and $\Sigma_{2}$ be smooth strictly convex compact disks of positive curvature in $\mathbf{R}^{3}$ which are tangent to each other at a common interior point $p$. Suppose that $\Sigma_{1}$ and $\Sigma_{2}$ lie locally on the same side of their common tangent plane at $p$, and that their intersection near $p$ consists of a finite number of smooth curve segments each of which emanates from $p$ in a distinct direction. Then for every open neighborhood $U_{1}$ of $p$ in $\Sigma_{1}$ there exists an open neighborhood $U_{2}$ of $p$ in $\Sigma_{2}$ and a smooth positively curved compact disk $\Sigma$ such that $\left(\Sigma_{1}-U_{1}\right) \cup U_{2} \subset \Sigma$, and $\Sigma$ contains $\Sigma_{1} \cap \Sigma_{2}$.

Step 3. We take the top loop from the figure-eight curve $E_{0} \cap S$ and merge it with the equator $\Gamma_{0}$ of $S$ to obtain a smooth simple closed curve $\Gamma_{1} \subset S$, as shown in the middle and right of Figure 2, See also Figure 3. Here the shaded region indicates the neighborhood $U$ mentioned in Step 2 above. The important point here is that $\Gamma_{1}$ contain a portion of the top loop of the figure-eight which begins and ends inside $U$.
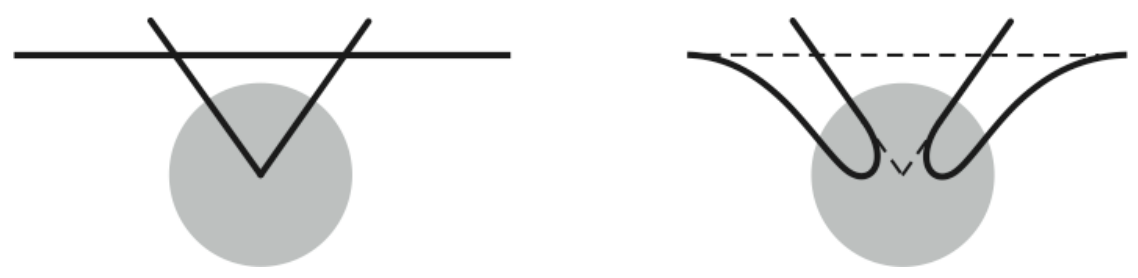

FIGURE 3.

Step 4. Now take a sequence of points $p_{0}, p_{1}, \ldots$ on the equator $\Gamma_{0}$ of $S$ which converge monotonically to a point $p_{\infty}$ of $\Gamma_{0}$ with respect to the intrinsic distance in $\Gamma_{0}$. Let $r_{0}, r_{1}, \ldots$ be a corresponding sequence of positive numbers so that the closures $B_{r_{i}}\left(p_{i}\right)$ are pairwise disjoint. Then repeating the first three steps, we obtain a sequence of smooth simple closed curves $\Gamma_{i} \subset S$. This sequence will converge, with respect to the Hausdorff metric, to a rectifiable curve $\Gamma_{\infty}$ which is differentiable everywhere, with respect to an arclength parametrization, and is smooth on $\Gamma_{\infty}-$ $\left\{p_{\infty}\right\}$, provided that $r_{i}$ converge to zero at a sufficiently fast rate. In particular, we need to choose $r_{i}$ so small that $B_{r_{i}}\left(p_{i}\right)$ is disjoint from a pinched solid torus $T$ which wraps around $\Gamma_{0}$ at $p_{\infty}$, see Figure 4, such that the interior of $T$ is disjoint from $\Gamma_{0}$ (since $\Gamma_{0}$ is smooth, such a torus exists at any point of it). By a pinched solid torus we mean the set of points in $\mathbf{R}^{3}$ whose distance from a circle of radius $r$ is at most $r$.

Step 5. By Jordan's curve theorem, $\Gamma_{\infty}$ divides $S$ into a pair of disks. Let $D$ be the bottom disk, see Figure 5. Now, using the same procedure described in Steps 1 and 2, we may glue to $D$ a piece of an ellipsoid, as depicted in Figure 5. Reflecting the boundary of the resulting surface with respect the $x y$-plane yields the desired curve $\Gamma$, which already bounds a smooth positively curved surface $M_{0}$ with positive curvature. Further, note that at the neck of each corrugation we have an open neighborhood where $M_{0}$ is tangent to an ellipsoid of revolution which contains 


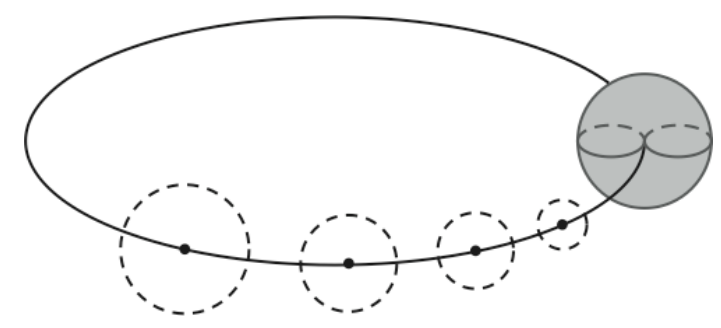

FiguRe 4.
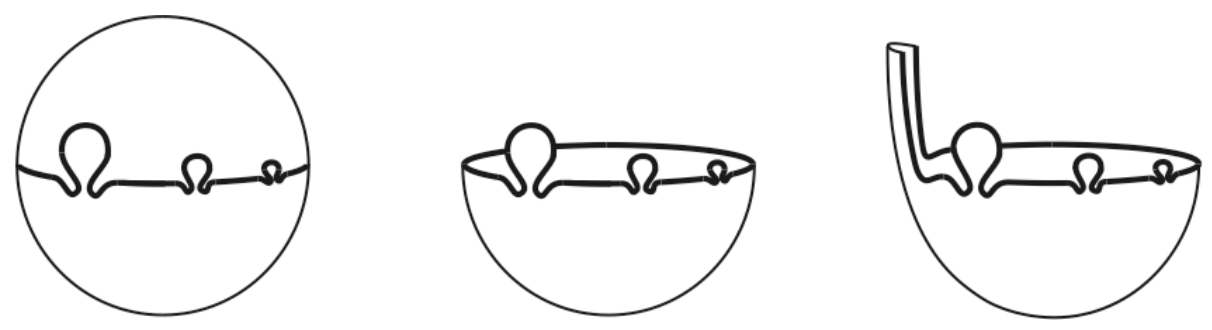

FIGURE 5.

the corrugation. So we may delete a pair of disks from $M_{0}$ which are bounded in part by a pair of corrugations and replace them with an annular piece of an ellipsoid. Thus we may construct a sequence of positively curved surfaces $M_{i}$ with $g\left(M_{i}\right)=i$ bounded by $\Gamma$. Note that each $M_{i}$ is smooth because, by construction, each boundary point of $M_{i}$ has an open neighborhood in $M_{i}$ which is part of an open neighborhood of a sphere or a slightly deformed ellipsoid.

2.3. Proof of Theorem 2.1. The basic plan for the proof of Theorem 2.1 is to take a small disk around $p$ in one of the surfaces, say $\Sigma_{2}$, and an annular neighborhood of the other surface $\Sigma_{1}$ surrounding the disk, see Figure 6, and then join the disk and the annulus to obtain a smooth positively curved surface containing the intersection curves of $\Sigma_{1} \cap \Sigma_{2}$ which run between the disk and the annulus. This is achieved by first building some thin "bridges" which connect the disk and the annulus along the intersection curves, see Proposition 2.7. Once these bridges are constructed, then the rest of the surface may be quickly filled in via Lemma 2.8, which is a result from 14. Our main task then is to establish the bridge principal stated in Proposition 2.7. whose proof is divided into the following lemmas.

Lemma 2.2. The space of positive definite $n \times n$ matrices is convex, i.e., if $A$ and $B$ are positive definite $n \times n$ matrices, then

$$
C:=\lambda A+(1-\lambda) B
$$

is also positive definite for all $0 \leq \lambda \leq 1$. 
Proof. A matrix $A$ is positive definite if and only if the associated quadratic form $Q_{A}(v):=v^{T} A v$ is positive definite. Further note that $Q_{C}(v)=\lambda Q_{A}(v)+(1-$ $\lambda) Q_{B}(v)$. Thus if $Q_{A}$ and $Q_{B}$ are positive definite, then so is $Q_{C}$.

Lemma 2.3. Let $U \subset \mathbf{R}^{n}$ be an open subset, $f, g, \phi: U \rightarrow \mathbf{R}$ be $\mathcal{C}^{2}$ functions, and set

$$
h:=\phi f+(1-\phi) g
$$

Suppose that $f(p)=g(p)$ and $\operatorname{grad} f(p)=\operatorname{grad} g(p)$ at some point $p \in U$. Then

$$
\text { Hess } h(p)=\phi(p) \text { Hess } f(p)+(1-\phi(p)) \text { Hess } g(p) \text {. }
$$

In particular, if $f$ and $g$ have positive definite Hessians at $p$, and $0 \leq \phi(p) \leq 1$, then $h$ has positive definite Hessian at $p$ as well.

Proof. Noting that $h=g+\phi(f-g)$, we easily compute the second partial derivatives

$$
h_{i j}=g_{i j}+\phi_{i j}(f-g)+\phi_{i}\left(f_{j}-g_{j}\right)+\phi\left(f_{i j}-g_{i j}\right) .
$$

Thus, since $f(p)=g(p)$ and $f_{i}(p)=g_{i}(p)$, it follows that

$$
h_{i j}(p)=g_{i j}(p)+\phi(p)\left(f_{i j}(p)-g_{i j}(p)\right)
$$

which completes the proof.

Let $B_{r}^{n}$ denote an open ball of radius $r$ centered at the origin of $\mathbf{R}^{n}$. Closure will be denoted by cl.

Lemma 2.4. Let $f, g: \operatorname{cl}\left(B_{r}^{2}\right) \rightarrow \mathbf{R}$ be $\mathcal{C}^{k \geq 2}$ functions with positive definite Hessians, and $\Gamma \subset \operatorname{cl}\left(B_{r}^{2}\right)$ be a connected curve which meets $\partial B_{r}^{2}$ only at its boundary points $\partial \Gamma=\left\{\left(x_{1}, y_{1}\right),\left(x_{2}, y_{2}\right)\right\}$. Suppose that $x_{1}<0, x_{2}>0$, and $f(p)=g(p)$, $\operatorname{grad} f(p)=\operatorname{grad} g(p)$ for all $p \in \Gamma$. Then there exists an open neighborhood $U$ of $\Gamma$ in $\operatorname{cl}\left(B_{r}^{2}\right)$, an $\epsilon>0$, and a $\mathcal{C}^{k}$ function $h: U \rightarrow \mathbf{R}$ with positive definite Hessian such that $f(p)=h(p)=g(p)$ for all $p \in \Gamma, h(x, y)=f(x, y)$ when $x \leq x_{1}+\epsilon$, and $h(x, y)=g(x, y)$ when $x \geq x_{2}-\epsilon$.

Proof. Choose $\epsilon$ so that $x_{1}+\epsilon<x_{2}-\epsilon$. Then there exists a smooth function $\bar{\phi}: \mathbf{R} \rightarrow \mathbf{R}$ such that $0 \leq \bar{\phi} \leq 1, \bar{\phi}=1$ when $x \leq x_{1}+\epsilon$, and $\bar{\phi}=0$ when $x \geq x_{2}-\epsilon$. Now define $\phi: \mathbf{R}^{2} \rightarrow \mathbf{R}$ by $\phi(x, y):=\bar{\phi}(x)$ and set $h:=\phi f+(1-\phi) g$.

Lemma 2.5. Let $\Gamma \subset \mathbf{R}^{3}$ be a compact connected embedded curve with endpoints $\partial \Gamma=\{p, q\}$. Suppose that there exist $\mathcal{C}^{k}$ positively curved surfaces $M_{i} \subset \mathbf{R}^{3}, i=$ $1, \ldots, n$ which cover $\Gamma$, with $p \subset M_{1}, q \subset M_{n}$, such that each $\Gamma_{i}:=M_{i} \cap \Gamma$ is a connected nonempty open subset of $\Gamma$, and whenever $\Gamma_{i} \cap \Gamma_{j} \neq \emptyset$, there exists an open neighborhood $U_{i j} \subset \Gamma_{i} \cap \Gamma_{j}$ where $M_{i}$ and $M_{j}$ are tangent for all $p \in U_{i j}$. Then there exists a $\mathcal{C}^{k}$ connected positively curved surface $M \subset \mathbf{R}^{3}$ which contains $\Gamma$. Further we may require that $M$ contain an open neighborhood of $p$ in $M_{1}$ and an open neighborhood of $q$ in $M_{n}$.

Proof. After replacing $M_{i}$ by a subcollection and reindexing we may assume that no subcollection of $\Gamma_{i}$ covers $\Gamma$, and $\Gamma_{i} \cap \Gamma_{j} \neq \emptyset$ if and only if $j=i \pm 1$. Then for $i=1, \ldots, n-1$ there exists a connected open neighborhood $U_{i} \subset \Gamma_{i} \cap \Gamma_{i+1}$ where $M_{i}$ and $M_{i+1}$ are tangent. Now take a point $p_{i} \in U_{i}$. Then in a neighborhood of $p_{i}$, 
$M_{i}$ and $M_{i+1}$ may be represented as the graphs of functions with positive definite Hessians over a small ball centered at $p_{i}$ in the tangent plane $T_{p_{i}} M_{i}=T_{p_{i}} M_{i+1}$. Then using the previous lemma, we may smoothly glue $M_{i}$ and $M_{i+1}$ near $p_{i}$. Then if we let $\bar{M}_{i}$ be small enough open neighborhoods (or strips) of $\Gamma$ in $M_{i}, M:=\cup_{i} \bar{M}_{i}$ is the desired surface.

We say that a $\mathcal{C}^{k \geq 2}$ embedded curve $\Gamma \subset \mathbf{R}^{3}$ is strictly convex if $\Gamma$ has nonvanishing curvature and through every point $p \in \Gamma$ there passes a strict support plane $\Pi$, i.e., $\Gamma$ lies on one side of $\Pi$ and intersects $\Pi$ only at $p$. By a nonsingular support vector field $n: \Gamma \rightarrow \mathbf{S}^{2}$ we mean a vector field such that if $\Pi_{p, n}$ denotes the plane which passes through $p$ and is orthogonal to $n$, then $\Pi_{p, n}$ is a strict support plane of $\Gamma$ at $p$, and furthermore, $n(p)$ is not orthogonal to the principal normal of $\Gamma$ at $p$.

Lemma 2.6 ([14]). Let $\Gamma \subset \mathbf{R}^{3}$ be a compact $\mathcal{C}^{k}$ strictly convex curve. Then $\Gamma$ lies on a $\mathcal{C}^{k}$ surface $M$ of positive curvature without boundary. Furthermore, for any $\mathcal{C}^{k}$ nonsingular support vector field $n: \Gamma \rightarrow \mathbf{S}^{2}$ along $\Gamma$ we may require that $M$ be normal to $n$.

Combining the last two lemmas now immediately yields the following bridge principal for positively curved surfaces:

Proposition 2.7. Let $M \subset \mathbf{R}^{3}$ be a $\mathcal{C}^{k}$ compact (but not necessarily connected) embedded surface of positive curvature, and $\Gamma \subset \mathbf{R}^{3}$ be a connected locally strictly convex curve segment with $\partial \Gamma=\left\{p_{1}, p_{2}\right\} \subset \partial M, p_{1} \neq p_{2}$. Suppose that $\Gamma$ meets $\partial M$ transversally, and that a neighborhood in $\Gamma$ of each $p_{i}$ lies in a local extension of $M$ at $p_{i}$. Then there exists a $\mathcal{C}^{k}$ compact surface of positive curvature $\bar{M}$ which contains $M$ and $\Gamma$. Further, if $n: \Gamma \rightarrow \mathbf{S}^{2}$ is a local nonsingular $\mathcal{C}^{k}$ support vector field along $\Gamma$, which is orthogonal to the local extensions of $M$ at $p_{i}$, then we may require that $\bar{M}$ be orthogonal to $n$ along $\Gamma$.

We only need to recall one more result from [14].

Lemma 2.8 ([14]). Every compact (but not necessarily connected) strictly convex $\mathcal{C}^{k}$ surface of positive curvature $\Sigma \subset \mathbf{R}^{3}$ lies on a $\mathcal{C}^{k}$ closed surface of positive curvature.

Using the last two results, we may now prove the main result of this section.

Proof of Theorem 2.1. Let us denote the intersection curves which emanate from $p$ and are contained in a small neighborhood of $p$ by $\Gamma_{i}, i=1, \ldots, k$. Since each $\Gamma_{i}$ is $\mathcal{C}^{1}$ and has a distinct tangent at $p$, we may assume that the given neighborhood $U_{1}$ of $p$ in $\Sigma_{1}$ is so small that $\Gamma_{i}$ and $\Gamma_{j}$ intersect each other in $U_{1}$ only at $p$ for $i \neq j$, each $\Gamma_{i} \cap U_{1}$ is connected, and if we parametrize each $\Gamma_{i} \cap U_{1}$ by $\gamma_{i}(t), t \in\left[0, \epsilon_{i}\right)$, $\gamma_{i}(0)=p$, then the distance between $\gamma_{i}(t)$ and $p$ is an increasing function of $t$. Now let $U_{2}$ be an open neighborhood of $p$ in $\Sigma_{2}$ which is chosen so small that $U_{2} \cap \Gamma_{i}$ is connected, and $\left(\Sigma_{1}-U_{1}\right) \cup \operatorname{cl}\left(U_{2}\right)$ is strictly convex. Note that $\Sigma_{1}-U_{1}$ and $\operatorname{cl}\left(U_{2}\right)$ are connected by a portion $\widetilde{\Gamma}_{i}$ of $\Gamma_{i}$, see Figure 6 . Using the bridge principle mentioned in the above proposition we may connect $\Sigma_{1}-U_{1}$ and $\mathrm{cl}\left(U_{2}\right)$ by thin strips of positive curvature containing $\widetilde{\Gamma}_{i}$ to obtain a compact positively curved surface $\widetilde{\Sigma}$ with $k+1$ 


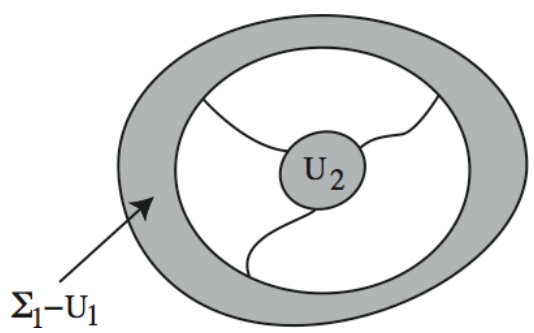

FiguRE 6.

boundary components (including $\partial \Sigma_{1}$ ). We can make sure that $\widetilde{\Sigma}$ is strictly convex by prescribing appropriate nonsingular support vector fields along each $\widetilde{\Gamma}_{i}$. Then by the previous lemma, $\widetilde{\Sigma}$ may be extended to a closed surface of positive curvature $O$. By the Jordan curve theorem, $\partial \Sigma_{1}$ divides $O$ into two components. The component which contains $\widetilde{\Sigma}$ is our desired surface $\Sigma$.

\section{Spanning Surfaces}

In this section we prove Theorems 1.2 and 1.3 , which concern two-dimensional fillings. Theorem 1.2 gives finiteness of topological types for the class of locally convex, compact spanning surfaces in $\mathbf{R}^{3}$, under minimal regularity assumptions. Here we use the theory of two-dimensional manifolds of bounded curvature due to Alexandrov and Zalgaller. Under greater regularity, Theorem 1.3 offers a far stronger finiteness statement for possibly noncompact spanning surfaces immersed in any given Riemannian manifold and having a uniform bound below on total curvature. This proof draws on classical results of Cohn-Vossen and Huber.

3.1. Proof of Theorem 1.2. Now we prove the finiteness result for the class $\mathcal{F}_{\Gamma}$ of compact, immersed, locally convex spanning surfaces of a finite collection $\Gamma$ of closed curves of finite turn immersed in $\mathbf{R}^{3}$.

The turn of a curve in $\mathbf{R}^{3}$ (also called the spatial turn) is the supremum of

$$
\Sigma\left(\pi-\alpha_{i}^{\prime}\right)
$$

over inscribed Euclidean polygons, where the $\alpha_{i}^{\prime}$ are the Euclidean angles at the vertices. See [5, Ch. V] for a discussion of the properties of curves of finite spatial turn. In particular, a curve of finite spatial turn has one-sided tangents and hence is rectifiable, and its turn is equal to the length of its tangent indicatrix on the unit sphere.

A locally convex surface without boundary is an example of a two-dimensional manifold of bounded curvature. These spaces were studied by Alexandrov and Zalgaller in [6], which is our basic reference throughout this section. The class consists of, besides two-dimensional Riemannian spaces, all two-dimensional metrized manifolds $M$ whose metric in a neighborhood of each point may be uniformly approximated by Riemannian metrics such that the integrals of the absolute values of the Gaussian curvatures are uniformly bounded. Equivalently, one can use polyhedral 
metrics for the approximation. The intrinsic defining property is that every point has a neighborhood in which the sum of positive excesses of nonoverlapping triangles is bounded. In this setting, the positive part $\omega^{+}$of curvature, and the negative part $\omega^{-}$, are defined as nonnegative, completely additive Borel set functions on $M$, and the curvature $\omega=\omega^{+}-\omega^{-}$is defined for any set for which at least one of $\omega^{+}$and $\omega^{-}$is finite. In the case of locally convex surfaces, $\omega^{-}=0$ and so $\omega$ is nonnegative.

The righthand turn of a geodesic polygon in $M$ is

$$
\Sigma\left(\pi-\alpha_{i}\right),
$$

where the $\alpha_{i}$ are the righthand sector angles between the directions at the interior vertices. Now consider a curve in $M$ with one-sided tangents at each point. The righthand turn of any open subarc $\gamma$ of this curve is the limit of the righthand turns of simple polygons in $D$ with the same endpoints as $\gamma$, converging to $\gamma$ and disjoint from $\gamma$ except at the endpoints, and whose angles with $\gamma$ at the endpoints converge to 0 . Under sufficient regularity, the righthand turn is equal to $\int \kappa_{g} d s$, where $\kappa_{g}$ is the geodesic curvature relative to the righthand normal. For a trivial subarc, namely, a single interior point, the turn is defined as $(\pi-\alpha)$ where $\alpha$ is the righthand sector angle between the directions at that point; when an endpoint is adjoined to $\gamma$, this quantity is added to the turn. The turn is additive under decomposition of a curve into disjoint subarcs, and is said to have bounded variation if, for a curve parameter $0 \leq t \leq 1$, the turn on the interval $(0, t)$ has bounded variation as a function of $t$.

The righthand turn of a curve $\gamma$ in a convex surface $M$ in $\mathbf{R}^{3}$ is bounded above in absolute value by the spatial turn of $\gamma$, as is the variation of righthand turn of $\gamma$. This fact is well-known in the Alexandrov-Pogorelov-Zalgaller theory of convex surfaces (see, for example, [9, p. 42]), but the argument is less well-known in the literature in English. The main idea (as described in [36, p.1045]) is that it suffices by approximation to consider a polygonal curve $\gamma_{k}$ in a convex polyhedron $M_{k}$. Then the variation of righthand turn of $\gamma_{k}$ in $M_{k}$ is at most equal to the variation of spatial turn of $\gamma_{k}$ because angles in $M_{k}$ are no smaller than spatial angles. And since the spatial turn restricted to a parameter interval $(0, t)$ is monotone in $t$, the variation of spatial turn equals the spatial turn.

With this preparation, we are ready to prove our finiteness theorem for locally convex surfaces $M$ that span a given collection $\Gamma_{i}, 1 \leq i \leq l$ of curves of finite turn:

Proof of Theorem 1.2. Denote the spatial turn of $\Gamma_{i}$ by $\kappa\left(\Gamma_{i}\right)$. For any locally convex spanning surface $M$, denote the one-sided turn of $\Gamma_{i}$ in $M$ by $\kappa_{M}\left(\Gamma_{i}\right)$. Since for a given polygonal arc in $M$, inscribed angles in $\mathbf{R}^{3}$ are less than the corresponding angles in $M$, there results $\left|\kappa_{M}\left(\Gamma_{i}\right)\right| \leq \kappa\left(\Gamma_{i}\right)$, so

$$
\kappa_{M}\left(\Gamma_{i}\right) \geq-\kappa\left(\Gamma_{i}\right) .
$$

By the Gauss-Bonnet theorem [6, p.192], and since $\omega(M) \geq 0$,

$$
2 \pi \chi(M)=\omega(M)+\sum_{i=1}^{l} \kappa_{M}\left(\Gamma_{i}\right) \geq-\sum_{i=1}^{l} \kappa\left(\Gamma_{i}\right) .
$$


Thus the Euler characteristics of all such spanning surfaces $M$ are uniformly bounded, and there are only finitely many possible topological types for $M$.

The observation about turn in the preceding proof has a smooth analogue which will be used several times in this paper:

Lemma 3.1. Suppose $\Gamma$ is the $\mathcal{C}^{2}$ boundary of a submanifold $M$ of a Riemannian manifold $N$. Then the second fundamental forms $\mathrm{II}_{\Gamma \hookrightarrow M}$ and $\mathrm{II}_{\Gamma \hookrightarrow N}$ of $\Gamma$ in $M$ and $\Gamma$ in $N$, respectively, satisfy $\left|\mathrm{II}_{\Gamma \hookrightarrow M}\right| \leq\left|\mathrm{II}_{\Gamma \hookrightarrow N}\right|$ pointwise.

The lemma follows directly from considering the projection of the $N$-covariant derivative of a geodesic direction in $\Gamma$ onto $T M$. Recall that $\mathrm{II}_{\Gamma \hookrightarrow M}$ assigns a bilinear form to each element of the unit normal bundle of $\Gamma$ in $M$, and similarly for $N .\left|\mathrm{II}_{\Gamma \hookrightarrow M}\right|$ and $\left|\mathrm{II}_{\Gamma \hookrightarrow N}\right|$ denote, respectively, the maximum of the norms of those bilinear forms (i.e. the largest of the absolute values of the eigenvalues of the associated quadratic forms).

What makes the lemma useful is that when $N$ and $\Gamma$ are considered fixed one gets a uniform bound on $\left|\mathrm{II}_{\Gamma \hookrightarrow M}\right|$.

3.2. Proof of Theorem 1.3. Here we show that a finite collection of closed $\mathcal{C}^{3}$ curves immersed in a given Riemannian manifold bounds at most finitely many topologically distinct, complete immersed $\mathcal{C}^{3}$ surfaces whose total curvature is uniformly bounded below. The proof follows from classical results of Cohn-Vossen and Huber on Gauss-Bonnet type theorems for noncompact surfaces, which have been proved in greater generality in [33, p. 46].

A surface is said to be finitely connected if it is homeomorphic to a compact surface minus finitely many points; otherwise, it is infinitely connected. We say that a smooth immersed surface is (metrically) complete if it is a complete metric space with respect to the induced metric, i.e., the Cauchy sequences in the surface converge (this is not to be confused with the other notion of completeness in Riemannian geometry which is concerned with extendibility of geodesics).

Lemma 3.2 (Cohn-Vossen). Let $M$ be a connected, finitely connected complete 2dimensional Riemannian manifold. Suppose that $\int_{M} K$ exists in $[-\infty, \infty]$, and $\partial M$ is compact. Then

$$
\int_{M} K+\int_{\partial M} \kappa_{g} \leq 2 \pi \chi(M)
$$

Lemma 3.3 (Huber). Let $M$ be a connected, infinitely connected, complete 2dimensional Riemannian manifold without boundary. Suppose that $\int_{M} K$ exists in $[-\infty, \infty]$. Then

$$
\int_{M} K=-\infty
$$

These results yield:

Proposition 3.4. Let $\mathcal{F}$ be a family of complete 2-dimensional manifolds $M$ with compact boundary, where the total curvature of $M$ and total geodesic curvature of $\partial M$ are both uniformly bounded below. Then $\mathcal{F}$ contains only finitely many topological types. 
Proof. For $M \in \mathcal{F}$, let $D(M)$ be the doubling of $M$ across its boundary, and $\widetilde{D}(M)$ be a smoothing of $D(M)$ which perturbs the metric only in a compact neighborhood of the boundary $\partial M$. Then the total curvature of $\widetilde{D}(M)$ is bounded below because it is bounded below on the complement of a compact set. Thus by Lemma 3.3. $\widetilde{D}(M)$ is finitely connected. So $M$ is finitely connected as well. Now Lemma 3.2 applies, and implies that $\chi(M)$ is bounded below. By the formulas for the Euler characteristic of a finitely connected surface, $M$ may assume only finitely many topological types.

To complete the proof of Theorem 1.3 , we only need to show then that the total geodesic curvature of $\partial M$ is bounded below. But note that if $M$ sits inside an ambient manifold $\bar{M}$ and $\kappa_{g}$ and $\bar{\kappa}_{g}$ denote the geodesic curvatures of $\partial M$ in $M$ and $\bar{M}$ respectively, then, $\kappa_{g} \geq-\bar{\kappa}_{g}$ by Lemma 3.1 It only remains to note that $\bar{\kappa}_{g}$ depends just on $\partial M$ which is fixed in $\bar{M}$, and thus $\int_{\partial M} \kappa_{g}$ is uniformly bounded below.

\section{Compact Higher-Dimensional Fillings}

This section considers finiteness of topological types for the class of compact fillings of dimension $n>2$. Here our theorems will be proved by using the theory of Alexandrov spaces of curvature bounded below.

4.1. Alexandrov spaces and finiteness. By a geodesic, we mean a distancerealizing path parametrized by arclength. All our spanning hypersurfaces are geodesic metric spaces in their intrinsic metrics, that is, every two points are joined by a geodesic.

Recall that a geodesic metric space is an Alexandrov space of curvature $\geq K$ if geodesic triangles are wider than comparison triangles in the simply connected space form $S_{K}$ of constant curvature $K$. Specifically, for any geodesic triangle $\triangle$ of perimeter $<2 \pi / \sqrt{K}$, the distance from a vertex to a point on the opposite side is at least the distance between corresponding points on a geodesic triangle with the same sidelengths in $S_{K}$. (Here the perimeter bound ensures that the model triangle is confined to an open hemisphere, and hence has a distinguished interior.) It follows that angles between geodesics may be defined, and the angle at each vertex of $\triangle$ is no less than the corresponding angle of the model triangle. (See [8] for a discussion.)

A complete Riemannian manifold $M$ with boundary is an Alexandrov space of curvature bounded below if and only if its interior sectional curvatures are bounded below and its boundary is locally convex. Any nonconvexity of the boundary produces branching of geodesics of the metric space $M$, and hence produces infinite negative curvature in the form of triangles that do not satisfy the wideness condition for any $K$.

There is a deep relation between Alexandrov spaces of curvature bounded below and finiteness of topological types. Here are the fundamental theorems: 
Theorem 4.1 (Gromov Compactness). Let $\mathcal{M}=\mathcal{M}(K, n, V, D)$ denote the class of Alexandrov spaces of curvature $\geq K$, dim $=n$, vol $\geq V$, and diam $\leq D$, where $\mathcal{M}$ carries the Gromov-Hausdorff metric. Then $\mathcal{M}$ is compact.

Theorem 4.2 (Perelman Stability, [28]). If $X \in \mathcal{M}$, then any $Y \in \mathcal{M}$ sufficiently close to $X$ is homeomorphic to $X$.

For a discussion of Gromov Compactness, one may consult [8, §10.7]. A simplified proof of Perelman's Stability Theorem is given in detail by Kapovitch [24. As an immediate consequence of Theorems 4.1 and $4.2, \mathcal{M}$ contains only finitely many topological types.

4.2. Proof of Theorem 1.4. We are going to exploit the regularity of the boundary submanifold $\Gamma$ in order to access this finiteness theory. The connection is via a warped product collar construction (Lemma 4.3) and Kosovskii's gluing theorem (Lemma 4.4). Lemma 4.3 allows the construction of a Riemannian collar $C=$ $\Gamma \times[0, T]$ such that $\Gamma \times\{T\}$ is totally geodesic, and $\Gamma \times\{0\}$ has a degree of convexity in $C$ that exceeds the concavity of $\Gamma$ in any $M$ in the filling family. Lemma 4.4 then allows all $M$ in the family to be extended to Alexandrov spaces with a uniform lower curvature bound.

Lemma 4.3 ([39, 40]). Suppose $\Gamma$ is any manifold (without boundary) having sectional curvature bounded below by $K_{1}$. Then for any $T>0$ and any $\lambda>0$, there exists a smooth metric on $C=\Gamma \times[0, T]$ such that $\mathrm{II}_{\Gamma \times\{0\}}-\lambda \mathrm{I}$ is positive definite, where $\mathrm{II}_{\Gamma \times\{0\}}$ is the second fundamental form of $\Gamma \times\{0\}$ in $C ; \Gamma \times\{0\}$ is isometric to $\Gamma ; \Gamma \times[T-\epsilon, T]$ is isometric to the direct product of $c \Gamma$ for some $0<c<1$ with the interval $[T-\epsilon, T]$; and the sectional curvature of $\Gamma \times[0, T]$ is bounded below by a constant $K=K\left(K_{1}, \lambda, T\right)$.

Typically, the lower bound $K$ will be strictly less than $K_{1}$.

Lemma 4.4 ([25]). Let $M_{1}$ and $M_{2}$ be two Riemannian manifolds-with-boundary, each having sectional curvatures bounded below by $K$, and whose boundaries are isometric and have respective second fundamental forms the sum of which is positive semidefinite. Then the space obtained by isometrically gluing $M_{1}$ to $M_{2}$ along their common boundary is an Alexandrov space of curvature bounded below by $K$.

Now we are ready to prove finiteness of topological types for the class of compact, smooth fillings with uniform bounds below on curvature and above on intrinsic diameter, of a compact immersed submanifold $\Gamma$ of arbitrary codimension in an arbitrary given Riemannian manifold $N$. Let $\mathcal{F}_{\Gamma}$ denote such a family of filling submanifolds with boundary $\Gamma$.

Proof of Theorem 1.4. Since $\Gamma$ is $\mathcal{C}^{2}$ smooth, the second fundamental form of $\Gamma$ in $N$ or in any $M \in \mathcal{F}_{\Gamma}$ is well-defined. Denote these forms by $\mathrm{II}_{\Gamma \hookrightarrow N}$ and $\mathrm{II}_{\Gamma \hookrightarrow M}$ respectively. For any $M \in \mathcal{F}_{\Gamma},\left|\mathrm{II}_{\Gamma \hookrightarrow M}\right| \leq\left|\mathrm{II}_{\Gamma \hookrightarrow N}\right|$ by Lemma 3.1.

Now we apply Lemma 4.3, with $\lambda:=\left|\mathrm{II}_{\Gamma \hookrightarrow N}\right|$, to obtain the collar $C=\Gamma \times[0, T]$. By construction, $C$ carries a metric such that $\Gamma \times\{0\}$ is isometric to $\Gamma$, and the sum of the second fundamental forms of $\Gamma \times\{0\}$ in $C$ and of $\Gamma$ in any $M \in \mathcal{F}_{\Gamma}$ is positive 
definite. Since all $M \in \mathcal{F}_{\Gamma}$ have the common boundary $\Gamma$, the same collar $C$ can be glued to each $M$. Let $K_{1}$ be a lower bound for the intrinsic sectional curvature of $\Gamma$. By isometrically gluing $C$ to $M$ along $\Gamma \times\{0\}$, using Lemma 4.4, one obtains an Alexandrov space $M \cup_{\Gamma} C$ of curvature bounded below by a uniform constant $K\left(K_{1}, \lambda, T\right)$.

Then the family $\left\{M \cup_{\Gamma} C: M \in \mathcal{F}_{\Gamma}\right\}$ has a uniform lower curvature bound, upper diameter bound and lower volume bound, and hence lies in a class $\mathcal{M}$ that contains only finitely many topological types. Since $M \cup_{\Gamma} C$ is homeomorphic to $M$, the class $\mathcal{F}_{\Gamma}$ itself admits only finitely many topological types.

Remark 4.5. As the preceding proof makes clear, Lemmas 4.3 and 4.4 imply the following intrinsic finiteness theorem for manifolds with boundary:

Theorem 4.6 ([39, 40]). There are only finitely many topological types of compact Riemannian n-manifolds with boundary having uniform bounds below on curvature and volume, and above on diameter and the norm of the second fundamental form of their boundaries.

4.3. Proof of Theorem 1.6. In this section, we consider a compact, immersed submanifold $\Gamma$ of codimension 2 in $\mathbf{R}^{n+1}$. Let $\mathcal{F}_{\Gamma}$ denote the family of all compact, nonnegatively curved, immersed hypersurfaces having $\Gamma$ as boundary. Since $\mathbf{R}^{n+1}$ is projectively equivalent to a hemisphere in $\mathbf{S}^{n+1}$, we may regard the spaces $M \in \mathcal{F}_{\Gamma}$ as hypersurfaces of sectional curvature $\geq 1$ in $\mathbf{S}^{n+1}$. Indeed, since projective maps preserve semidefiniteness of the second fundamental forms of hypersurfaces, this claim is a consequence of the Gauss Equation (see [13]). From now on in this subsection, we use the Riemannian metrics induced from $\mathbf{S}^{n+1}$.

Denote the components of $\Gamma$ by $\Gamma_{i}, 1 \leq i \leq l$. The following lemma bounds the intrinsic diameter of any $M \in \mathcal{F}_{\Gamma}$ in terms of the intrinsic diameters $\operatorname{diam}\left(\Gamma_{i}\right)$ of the $\Gamma_{i}$.

Lemma 4.7. The intrinsic diameter of $M \in \mathcal{F}_{\Gamma}$ is uniformly bounded above by

$$
(l+1) \pi+\Sigma_{i=1}^{l} \operatorname{diam}\left(\Gamma_{i}\right) .
$$

Proof. First note that the distance of any point in $M \in \mathcal{F}_{\Gamma}$ to $\Gamma$ is $\leq \pi$; for, otherwise, the distance from some $p \in M$ to $\Gamma$ would be realized by an $M$-geodesic $\gamma$ of length $>\pi$, and $\gamma$ would have a subsegment from $p$ of length $>\pi$ that does not contact $\Gamma$. Since $M$ has sectional curvature $\geq 1$, such a subsegment would contain an internal pair of conjugate points by the Rauch comparison theorem. Therefore the subsegment could be shortened by a variation with endpoints fixed in an arbitrarily small neighborhood of itself (see, for example, [34, p.316]), yielding a contradiction.

Now let $\gamma$ be a geodesic in $M$. The distance from the initial point to the first intersection with the boundary, say with $\Gamma_{1}$, is $\leq \pi$. The distance from the first intersection point to the last intersection point of $\gamma$ with $\Gamma_{1}$ is at most $\operatorname{diam}\left(\Gamma_{1}\right)$. Now reiterate this argument, starting from the last intersection point of $\gamma$ with $\Gamma_{1}$.

With this preparation, the proof of Theorem 1.6 is immediate: 
Proof of Theorem 1.6. The proof proceeds just as in the proof of Theorem 1.4, except that here we have not assumed an a priori bound on the diameters of the $M \in \mathcal{F}_{\Gamma}$, but rather, may invoke Lemma 4.7.

Remark 4.8. As we have just shown, Theorem 1.6 also holds in $\mathbf{S}^{n+1}$ for spanning hypersurfaces with curvature $\geq 1$. Similarly, by projective transformation, Theorem 1.6 holds in $\mathbf{H}^{n+1}$ for compact hypersurfaces with curvature $\geq-1$. The latter case is interesting for being a finiteness theorem where there is no uniform lower bound on total negative curvature.

4.4. Proof of Theorem 1.5. First we need a relative version of Nash's isometric embedding theorem. Let $0^{n}$ denote the origin of $\mathbf{R}^{n}$.

Theorem 4.9 ([16]). Let $(M, g)$ be a $\mathcal{C}^{\infty}$ Riemannian n-manifold, $p \in M$, and $U$ be a neighborhood of $p$. Suppose there exists a $\mathcal{C}^{\infty}$ isometric embedding $f: U \rightarrow$ $\mathbf{R}^{m}, m>n$. Then there exists a smooth embedding $\bar{f}: M \rightarrow \mathbf{R}^{N}$, where $N=$ $\max \{n(n+5) / 2, n(n+3) / 2+5\}+m$, and a neighborhood $V \subset U$ of $p$ such that $\left.\bar{f}\right|_{V}=f \times\left\{0^{N-m}\right\}$, and the pull-back metric $\bar{f}^{*}\langle$,$\rangle is arbitrarily close to g$, with respect to the $\mathcal{C}^{\infty}$ topology.

The above result quickly yields the desired example of infinitely many topologically distinct, positively curved 3-dimensional submanifolds spanning a common boundary in $\mathbf{R}^{18}$ :

Proof of Theorem 1.5. Let $M_{i}$ denote the lens spaces $\mathbf{S}^{3} / \mathbf{Z}_{i}, i=1,2, \ldots$, and choose $p_{i} \in M_{i}$. Since $M_{i}$ are locally isometric to $\mathbf{S}^{3} \subset \mathbf{R}^{4}$, there are, by Theorem 4.9 . approximate isometric embeddings $f_{i}: M_{i} \rightarrow \mathbf{R}^{14+4}$, such that for sufficiently small neighborhoods $V_{i}$ of $p_{i}$ we have $f_{i}\left(V_{i}\right) \subset \mathbf{S}^{3}$. Further note that $f_{i}$ have positive curvature, since $f_{i}^{*}\langle$,$\rangle are \mathcal{C}^{2}$ close to the original metric of $M_{i}$ which have positive curvature. Let $B_{\epsilon_{i}}(p) \subset M_{i}$ be open metric balls centered at $p_{i}$, such that $B_{\epsilon_{i}}(p) \subset$ $V_{i}$, and set $\bar{M}_{i}:=f_{i}\left(M_{i}-B_{\epsilon_{i}}(p)\right)$. Then $\partial \bar{M}_{i}$ will be geometric spheres. Indeed each $\partial \bar{M}_{i}$ can be made to coincide with $\mathbf{S}^{2} \subset \mathbf{R}^{3} \times\left\{0^{15}\right\}$ after a rigid motion and a homothety in $\mathbf{R}^{18}$. These transformations, applied to $\bar{M}_{i}$, yield our desired submanifolds, since $M_{i}$, and consequently $\bar{M}_{i}$, are pairwise nonhomeomorphic.

\section{Complete Higher-Dimensional Fillings}

5.1. Proof of Theorem 1.7. Finally we turn to the case of noncompact complete fillings. In this section, we again assume $n>2$, since we already have established Theorem 1.3 for surfaces. We fix a compact, immersed submanifold $\Gamma$ of codimension 2 in $\mathbf{R}^{n+1}$. Our goal is to show that $\Gamma$ is spanned by only finitely many topological types of complete, nonnegatively curved, immersed hypersurfaces whose second fundamental form has nullity at most 1 .

Our procedure is first to bound the number of ends of any nonnegatively curved hypersurface spanning $\Gamma$. Then we "clip off" the ends, so that the proof of Theorem 1.7 becomes similar to that of the compact case already considered in Section 4 .

The new elements we draw on here include a theorem of Cai that bounds the number of ends of a Riemannian manifold whose Ricci curvature is nonnegative off 
a compact set and everywhere bounded below (Lemma 5.1); a theorem of Perelman on intrinsic gluing and smoothing under a lower Ricci curvature bound (Lemma 5.2); and a theorem of Alexander and Currier on the end representatives of a complete Euclidean hypersurface that, except on some compact set, is nonnegatively curved with second fundamental form of nullity at most 1 (Lemma 5.4).

Specifically, Lemma 5.2 , combined with an argument involving collaring, doubling and surgery, allows us to invoke Lemma 5.1 to bound the number of ends. Lemma 5.4 specifies the topology of the ends. Now we may compactify the spanning hypersurfaces by a clipping procedure. Since these compactified fillings are no longer smooth, we need a singular Bonnet-Myers comparison (Lemma 5.5) in order to finish the proof.

By an end representative in a complete Riemannian manifold $M$, we mean an unbounded component of the complement of a compact subset of $M$. An end is an equivalence class of nested decreasing sequences of end representatives that eventually lie outside every compact subset of $M$, where two sequences are equivalent if any member of either contains a truncation of the other.

Lemma 5.1 ([11]). In a complete $n$-dimensional Riemannian manifold without boundary whose Ricci curvature satisfies ric $\geq 0$ off a metric ball of radius a and ric $>\Lambda$ everywhere, there is a universal bound $c(n, a, \Lambda)$ on the number of ends.

This control on the number of ends only requires lower Ricci bounds, and these behave well under gluing and smoothing, as the following theorem shows.

Lemma 5.2 ([30]). Let $N_{1}$ and $N_{2}$ be two Riemannian manifolds with isometric compact boundaries and $\operatorname{ric}\left(N_{i}\right)>\Lambda$. Suppose the sum of the second fundamental forms is positive definite at each point on the identified boundaries. Then the induced metric on the gluing $N_{1} \cup N_{2}$ can be smoothed in an arbitrarily small tubular neighborhood of the identified boundaries to yield a complete $\mathcal{C}^{2}$ manifold with ric $>\Lambda$.

This theorem was originally stated for positive Ricci curvature, but the proof holds for any lower bound. The proof described in [30] is written out in detail in an appendix of Wang's thesis [38].

Lemma 5.3. Given a compact submanifold $\Gamma$ of codimension 2 in $\mathbf{R}^{n+1}$, consider all complete, immersed, nonnegatively curved spanning hypersurfaces $M$ of $\Gamma$. Then the number of ends of $M$ is uniformly bounded.

Proof. Let $\left\{\Gamma_{1}, \ldots, \Gamma_{l}\right\}$ be the components of $\Gamma$. For any spanning hypersurface $M$, we consider the glued manifold $M \cup_{\Gamma} C$, where $C=\cup_{i=1}^{l} \Gamma_{i} \times[0, T]$ is a (fixed) collar which may be constructed, with the aid of Lemma 4.3 , similarly to the description in the proof of Theorem 1.4. In particular, setting $\lambda:=\left|\mathrm{II}_{\Gamma \hookrightarrow \mathbf{R}^{n+1}}\right|$, i.e. the norm of the second fundamental form of $\Gamma$ in $\mathbf{R}^{n+1}$, we can make sure that the sum of the second fundamental forms of $M$ and $C$ at the corresponding points of gluing across $\Gamma$ is positive definite.

Since $C$ is compact and the spanning hypersurfaces $M$ are nonnegatively curved, there is a uniform lower bound on the sectional curvatures of $C$ and the manifolds 
$M$, and consequently a uniform Ricci bound, ric $>\Lambda$. Therefore, by Lemma 5.2 , the metric of each $M \cup_{\Gamma} C$ may be smoothed arbitrarily close to $\Gamma$ while retaining ric $>\Lambda$. Denote the resulting manifold by $\widetilde{M}$. Since, by Lemma 4.3 , each $\widetilde{M}$ is cylindrical near its boundary $\Gamma \times\{T\}$, doubling across the boundary yields a complete, smooth Riemannian manifold $D(\widetilde{M})$ still satisfying ric $>\Lambda$.

Now note that removing the two copies of $\operatorname{int}(M)$ in $D(\widetilde{M})$ leaves $l$ components $D\left(C_{i}\right)$, where $C_{i}$ is a component of $C$ (namely, $\Gamma_{i} \times[0, T]$ carrying the restriction of the metric of $\widetilde{M})$ and $D\left(C_{i}\right)$ is the doubling of $C_{i}$ across $\Gamma_{i} \times\{T\}$. We may join $D\left(C_{i}\right)$ to $D\left(C_{i+1}\right)$, for $1 \leq i \leq k-1$, by a surgery procedure inside $D(C)$, i.e., we remove from the interiors of $D\left(C_{i}\right)$ and $D\left(C_{i+1}\right)$ a pair of small open balls, replace them by an annulus, and use a partition of unity to endow the gluing with a smooth metric which coincides with the metric on $D(C)$ near its boundaries. Thus these gluings transform $D(\widetilde{M})$ and $D(C)$ to smooth connected Riemannian manifolds $D(\widetilde{M})^{*}$ and $D(C)^{*}$ respectively, such that, for all $M \in \mathcal{F}_{\Gamma}, D(\widetilde{M})^{*}-D(C)^{*}=D(\widetilde{M})-D(C)$.

Since $D(C)^{*}$ is compact, it has a lower Ricci bound $\Lambda^{\prime}$ which does not depend on $M$. Further, since $D(C)^{*}$ is connected, its diameter $\Delta$ is well defined and yields a uniform upper bound for diameter of $D(C)^{*}$ in $D(\widetilde{M})^{*}$. Thus there exists a point $p$ of $D(C)^{*}$ such the metric ball $B$ in each $D(\widetilde{M})^{*}$ centered at $p$ and of uniform radius $\Delta+\epsilon$ for some $\epsilon>0$ contains an open neighborhood of $D(C)^{*}$. Outside of this neighborhood, we may assume $D(\widetilde{M})^{*}$ to have nonnegative curvature since, as described earlier in this proof when we invoked Lemma $5.2, \widetilde{M}$ coincides with $M$ outside of a uniformly small neighborhood of $C$. Thus $D(\widetilde{M})^{*}-B$ has nonnegative Ricci curvature, and $B$ has a uniform lower Ricci bound given by $\min \left\{\Lambda, \Lambda^{\prime}\right\}$.

By Lemma 5.1, it then follows that the number of ends of $D(\widetilde{M})^{*}$ is uniformly bounded. Therefore there is a uniform bound for the number of ends of $\widetilde{M}$, and hence of $M \in \mathcal{F}_{\Gamma}$, since $M$ and $\widetilde{M}$ are homeomorphic.

By a convex body in a Euclidean space we mean a closed convex subset with interior points; its boundary is a convex hypersurface. A convex cap is the intersection of a convex hypersurface with an open halfspace. The following theorem is proved in [4. The topological structure of the ends is implicit there; here we outline the proof in order to give a more detailed discussion of the topology.

Lemma 5.4. Let $M$ be a complete, smoothly immersed hypersurface without boundary of $\mathbf{R}^{n+1}, n>2$. Suppose there is a compact set $X$ in $M$ such that $M-X$ is nonnegatively curved and its second fundamental form has nullity at most 1 everywhere. Then each end of $M$ has a representative that is homeomorphic to $\mathbf{S}^{n-1} \times[0,1)$ and is embedded onto the union of two convex caps.

Outline of proof. The proof adapts the method of slicing with moving hyperplanes first used by van Heijenoort [37] (see [4, Thm. 2]). It also draws on the theorem of Sacksteder which states that a complete, immersed hypersurface of $\mathbf{R}^{n}$ with nonnegative but not identically zero sectional curvature is embedded as the boundary 
of a convex body [32, and that of Hartman and Nirenberg, that identically zero curvature only occurs for an $(n-2)$-cylinder over a plane curve [21].

Suppose $H \subset \mathbf{R}^{n+1}$ is a hyperplane through $p \in M$ that does not intersect $X$, and is transverse to $T_{p} M$ and the nullity subspace at $p$. Then by a transversality argument and Sacksteder's theorem, each component $N$, which is not a singleton, of the intersection of $M$ with $H$ is a convex hypersurface of $H$. For any point $p \in M$ outside a Euclidean ball containing $X$, slicing by rotated hyperplanes through $p$ produces a subset $C_{p}$ of $M$ that is embedded onto a convex cap, and has boundary that contains $p$ and intersects $X$ (see [4, Lemma 2]). An unbounded sequence of points $p_{i}$ of $M$ may be extracted lying outside a ball containing $X$, and the existence of convex caps $C_{p_{i}}$ together with the compactness of $X$ ensure that in each end there is a convex cap $C$ with noncompact closure that contains no line (see [4, Lemma $3])$.

If $\partial C$ is compact, then by definition $C$ is an end representative. That is, $C=$ $\partial K \cap \operatorname{int}\left(H^{+}\right)$, where $H^{+}$is a closed halfspace bounded by $H$, and $K \subset H^{+}$is an unbounded convex body in $\mathbf{R}^{n+1}$. We may assume that $K \cap H$ has interior points in $H$, after moving $H$ parallel to itself into int $\left(H^{+}\right)$. Thus $\partial C:=\operatorname{cl}(C)-C=\partial(K \cap H)$ is a convex hypersurface of $H$. Since $\partial C$ is compact, $\partial C$ is homeomorphic to $\mathbf{S}^{n-1}$. Consequently, the interior of $K \cap H$ is homeomorphic to an open ball $B^{n}$. Since $\operatorname{cl}(C)=\partial K-\operatorname{int}(K \cap H)$, it follows that $\operatorname{cl}(C)$ is homeomorphic to $\partial K$ minus an open ball. Since $\partial K$ is a noncompact complete convex hypersurface of $\mathbf{R}^{n+1}$ that contains no line, $\partial K$ is homeomorphic to $\mathbf{R}^{n}$, and so $\operatorname{cl}(C)$ is homeomorphic to $\mathbf{S}^{n-1} \times[0,1)$.

If $\partial C$ is noncompact, we proceed as follows. Since $C$ contains no line, the "recession directions" of the convex hull $\operatorname{conv}(C)$ point into an open halfspace of the form $\langle y, v\rangle>0$. Therefore the slices of $\operatorname{conv}(C)$ by hyperplanes $H_{t}:\langle y, v\rangle=t$ for $t \geq \alpha$ sufficiently large are compact. Denote by $S_{t}$ the extensions of these slices of $\operatorname{conv}(C)$ to slices of $M$, and set $C_{v}=\cup_{t>\alpha} S_{t}$. The theorems of Sacksteder, and Hartman and Nirenberg, imply that $S_{t}$ is a convex hypersurface of $H_{t}$, and so $C_{v}$ is a noncompact convex cap. If any of the extended slices are compact, then they are all compact and homeomorphic to $\mathbf{S}^{n-1}$. Then $C_{v}$ is an end representative whose closure is homeomorphic to $\mathbf{S}^{n-1} \times[0,1)$ and lies on the boundary of a convex body. Suppose on the other hand that the extended slices are noncompact. Then we may choose a recession direction $w$ for these slices, and consider slices $S_{t}^{\prime}$, meeting $C_{v}$, of $M$ by hyperplanes $H_{t}^{\prime}:\langle y, w\rangle=t$ for $t>\alpha$. Increasing $\alpha$ if necessary so that $H_{t}^{\prime}$ does not meet $X$ for $t \geq \alpha$, the $S_{t}^{\prime}$ are again convex hypersurfaces of the $H_{t}^{\prime}$ and we again have a noncompact convex cap $C_{w}=\cup_{t>\alpha} S_{t}^{\prime}$. Then $v$ is a recession direction for $C_{w}$; perturbing $v$ and $w$ if necessary by a slight rotation in the plane they determine, and invoking the nullity hypothesis, ensures in addition that $-v$ is not a recession direction for $C_{w}$. Therefore $T_{t}^{\prime}:=S_{t}^{\prime} \cap\{y:\langle y, v\rangle \leq t\}$ is compact and homeomorphic to a closed $(n-1)$-ball. The union $T_{t} \cup T_{t}^{\prime}$, where $T_{t}=S_{t} \cap\{y:\langle y, w\rangle \leq t\}$, is then homeomorphic to $\mathbf{S}^{n-1}$. Thus we obtain an end representative of the form $C_{v} \cup C_{w}=\cup_{t>\alpha}\left(T_{t} \cup T_{t}^{\prime}\right)$ (see [4, Proof of Theorem 1]). It is easily verified that 
there is a continuous map with a continuous inverse from the closure of this end representative onto $\mathbf{S}^{n-1} \times[0,1)$.

Now we are ready to prove our finiteness theorem for noncompact, complete fillings of a given compact submanifold $\Gamma$ of codimension 2 in $\mathbf{R}^{n+1}$, where $n>2$. Let $\mathcal{F}_{\Gamma}$ denote all complete, smoothly immersed spanning hypersurfaces of $\Gamma$ having nonnegative sectional curvature and second fundamental form of nullity at most 1 . We must show that $\mathcal{F}_{\Gamma}$ contains only finitely many topological types.

Proof of Theorem 1.7. Step 1. First we "clip off" the ends of $M$. When $M \in \mathcal{F}_{\Gamma}$ is noncompact, its projective image in $\mathbf{S}^{n+1}$ need not be complete, so this compactification step must precede any projective transformation step. Specifically, by Lemma 5.4. we obtain a compact, locally convex (but not smooth) spanning hypersurface $\bar{M}$ of $\Gamma$ by discarding, for each end, an embedded end representative. The discarded representative either is a convex cap $C_{v}$ that lies in a halfspace $H_{t}^{+}$and has boundary coinciding with the boundary of a compact convex body $B_{t}$ in the hyperplane $H_{t}$, or else is the union $C_{v} \cup C_{w}$ of two convex caps, lies in a union $H_{t}^{+} \cup H_{t}^{\prime+}$ of two orthogonal halfspaces, and has boundary coinciding with the boundary of a union $B_{t} \cup B_{t}^{\prime}$, where $B_{t}$ is a compact convex body in the hyperplane $H_{t}, B_{t}^{\prime}$ is a compact convex body in the hyperplane $H_{t}^{\prime}$ and

$$
B_{t} \cap \operatorname{int}\left(H_{t}^{\prime+}\right)=\emptyset, \quad B_{t}^{\prime} \cap \operatorname{int}\left(H_{t}^{+}\right)=\emptyset .
$$

The discarded end representative is replaced by gluing in $B_{t}$ in the first case, and $B_{t} \cup B_{t}^{\prime}$ in the second case, to obtain $\bar{M}$. Figure 7 illustrates this procedure when $n=2$.

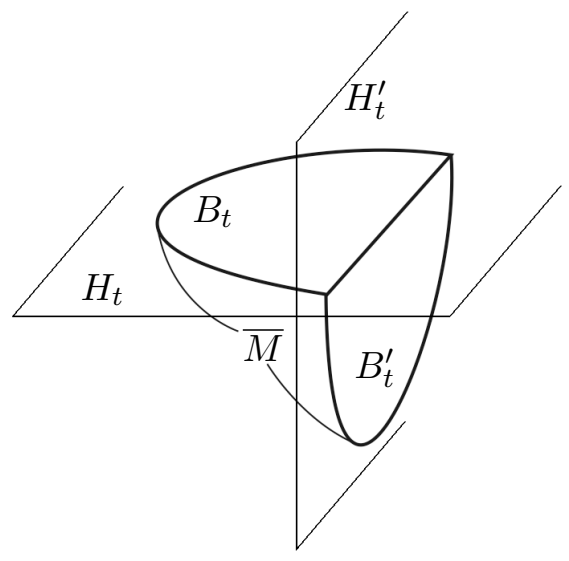

FiguRE 7.

We are going to prove finiteness of the topological types for the family

$$
\overline{\mathcal{F}}_{\Gamma}=\left\{\bar{M}: M \in \mathcal{F}_{\Gamma}\right\}
$$


of compactified spanning hypersurfaces. For this purpose, we modify the proofs of Theorems 1.4 and 1.6 to accommodate the reduced regularity of members of $\overline{\mathcal{F}}_{\Gamma}$.

Step 2. Regard each $\bar{M} \in \overline{\mathcal{F}}_{\Gamma}$ as a hypersurface in $\mathbf{S}^{n+1}$ by a projective transformation to a hemisphere, so that from now on $\bar{M}$ denotes a metric space carrying the intrinsic metric induced from $\mathbf{S}^{n+1}$. Since local convexity is a projectively invariant property, $\bar{M}$ is locally convex in $\mathbf{S}^{n+1}$. Thus the metric of $\bar{M}$ has Alexandrov curvature $\geq 1$ locally in the interior of $\bar{M}$ 3.

In $\$ 5.2$, we will prove the following Bonnet-Myers comparison lemma for $\bar{M} \in \overline{\mathcal{F}}_{\Gamma}$. It seems likely that this lemma holds for all local Alexandrov spaces of curvature $\geq 1$, not just for $\bar{M} \in \overline{\mathcal{F}}_{\Gamma}$, but a proof in the former case is not known and the technicalities in the latter case are comparatively mild.

Lemma 5.5. The distance of any point of $\bar{M} \in \overline{\mathcal{F}}_{\Gamma}$ to $\Gamma$ is not greater than $\pi$.

Step 3. Now we may proceed as in the proof of Theorem 1.4. There is a fixed collar $C$ such that for all $\bar{M} \in \overline{\mathcal{F}}_{\Gamma}$, the glued spaces $\bar{M} \cup_{\Gamma} C$ are Alexandrov spaces of curvature bounded below by a uniform constant. By virtue of Lemma 5.5, both the statement and the proof of Lemma 4.7 apply essentially unchanged to give a uniform bound on the intrinsic diameter of $\bar{M} \in \overline{\mathcal{F}}_{\Gamma}$. In consequence, the family $\left\{\bar{M} \cup_{\Gamma} C: \bar{M} \in \overline{\mathcal{F}}_{\Gamma}\right\}$ has uniform lower curvature bound, upper diameter bound and lower volume bound, and hence lies in a class $\mathcal{M}$ that contains only finitely many topological types. Since $\bar{M} \cup_{\Gamma} C$ is homeomorphic to $\bar{M}$, the class $\overline{\mathcal{F}}_{\Gamma}$ itself admits only finitely many topological types.

Moreover, by Lemma 5.1, the number of clipped ends is uniformly bounded, and by Lemma 5.4 the closure of each clipped end representative is homeomorphic to $\mathbf{S}^{n-1} \times[0,1)$. So it follows that $\mathcal{F}_{\Gamma}$ itself contains only finitely many homeomorphism classes. Here we have used the fact that any gluing of the end representative to the rest of the manifold is determined by a homeomorphism of $\mathbf{S}^{n-1}$ to itself, which is isotopic to the identity; thus, topologically speaking, there is only one way to glue the end representative back to the rest of $M$, which in turn yields the finiteness that we seek.

Remark 5.6. If Theorem 1.7 is restricted to strictly positively curved spanning hypersurfaces $M$, the proof may be simplified by appealing to known results, avoiding Lemma 5.5. In this case, it is shown in [4] that each end of $M \in \mathcal{F}_{\Gamma}$ may be replaced by gluing in a compact convex body $B_{t}$ lying in a hyperplane $H_{t}$. Then the main result of [14] may be used to show that the resulting $\bar{M}$ has a positively curved smoothing. Thus the theorem is reduced to the smooth compact case considered in \$4.

5.2. Proof of Lemma 5.5. In Step 1 of the proof of Theorem 1.7, a compact, locally convex hypersurface $\bar{M}$ is constructed in $\mathbf{R}^{n+1}$ by clipping off the ends of $M$. That is, we replace a certain end representative of each end of $M$ by a compact cap, namely, a compact set of the form $B \cup B^{\prime}$, where $B$ is a compact convex body in a hyperplane $H, B^{\prime}$ is a compact convex body in an orthogonal hyperplane $H^{\prime}$, and $B \cap \operatorname{int}\left(H^{\prime+}\right)=\emptyset, \quad B^{\prime} \cap \operatorname{int}\left(H^{+}\right)=\emptyset$. (It is possible that a single $B$ suffices. Since the argument there is simpler, we consider the general case here.) 
Since the number of ends is bounded by Lemma 5.3 , then there are finitely many of these compact caps, say $B_{i} \cup B_{i}^{\prime}, 1 \leq i \leq k$. By Step 2, we then regard each $\bar{M} \in \overline{\mathcal{F}}_{\Gamma}$ as a locally convex hypersurface in a given open hemisphere $\mathbf{S}$ of $\mathbf{S}^{n+1}$, so that the $B_{i}$ and $B_{i}^{\prime}$ become convex bodies in totally geodesic hyperspheres.

We use the following slightly nonstandard terminology adapted to our construction. The $n$-faces of $\bar{M}$ are the relative interiors of the compact caps and of the portion of $M$ that remains after clipping, respectively, namely

$$
\operatorname{int}\left(B_{i} \cup B_{i}^{\prime}\right), \operatorname{int}(M \cap \bar{M}),
$$

$1 \leq i \leq k$. An $n$-face $\operatorname{int}\left(B_{i} \cup B_{i}^{\prime}\right)$ is intrinsically isometric to an open subset of a totally geodesic hypersphere. The $(n-1)$-faces are

$$
\operatorname{int}\left(B_{i} \cap M\right)=\partial B_{i}-H^{\prime}, \operatorname{int}\left(B_{i}^{\prime} \cap M\right)=\partial B_{i}^{\prime}-H,
$$

and the $(n-2)$-faces are

$$
B_{i} \cap B_{i}^{\prime} \cap M=\partial\left(B_{i} \cap B_{i}^{\prime}\right)=\partial\left(B_{i} \cap M\right)=\partial\left(B_{i}^{\prime} \cap M\right) .
$$

The $(n-2)$-faces are smooth convex hypersurfaces that bound the convex bodies $B_{i} \cap B_{i}^{\prime}$ in the totally geodesic $(n-1)$-spheres determined by $H_{i} \cap H_{i}^{\prime}$.

In this setting, the basic constructions of Alexandrov geometry are fairly simple (see [8]): The direction space $U_{p} \bar{M}$ of $\bar{M}$ at any interior point $p$ is the metric space of unit tangent vectors at $p$ with the angle metric, while the tangent space $T_{p} \bar{M}$ is the linear cone over $U_{p} \bar{M}$. If $p$ is not on an $(n-2)$-face, then $T_{p} \bar{M}$ is isometric to $\mathbf{R}^{n}$. If $p$ lies on an $(n-2)$-face $F$, then $T_{p} \bar{M}$ bounds the intersection of three transverse halfspaces through the origin, and hence is the metric product of a Euclidean factor $\mathbf{R}^{n-2}$ with the cone over a homeomorph of a circle obtained by gluing together three circular arcs of radius 1 , the sum of whose lengths is less than $2 \pi$. (The intersection of $\bar{M}$ with the 3 -plane orthogonal to $F$ at $p$ has a neighborhood as shown around a corner point in Figure 7.) Furthermore, any geodesic in int $\bar{M}$ that has an internal point on an $(n-2)$-face $F$ must be tangent to the $\mathbf{R}^{n-2}$ factor there [8, p. 109].

Suppose $\gamma$ is a geodesic in int $\bar{M}$. By the convexity of $B_{i}$, the shortest path in the hemisphere $\mathbf{S}$ and hence in $\bar{M}$ between two points of $B_{i}$ lies in $B_{i}$, and similarly for $B_{i}^{\prime}$. Therefore $\gamma \cap\left(B_{i} \cup B_{i}^{\prime}\right)$ has zero, one or two components; we call a nonempty component a clipping segment. Then $\gamma$ is a disjoint union of clipping segments and nontrivial geodesic segments of $\operatorname{int}(M \cap \bar{M})$, which alternate.

Lemma 5.7. Suppose $\gamma$ is a geodesic of $\bar{M}$ such that if $\gamma$ has an endpoint in an $(n-2)$-face, then a nontrivial end subsegment of $\gamma$ lies in that face. Then any clipping segment $\sigma$ of $\gamma$ either lies in an $n$-face $\operatorname{int}\left(B_{i} \cup B_{i}^{\prime}\right)$, except possibly for endpoints at which $\sigma$ strikes an $(n-1)$-face transversely, or else lies in a lowerdimensional face.

Proof. Suppose the clipping segment $\sigma$ contains a point $p$ on a lower-dimensional face $F$. If $F$ is an $(n-2)$-face, then by assumption, $p$ may be taken interior to $\sigma$. Then $\sigma$ must be tangent to $F=\partial\left(B_{i} \cap B_{i}^{\prime}\right)$ at $p$. By convexity of $B_{i} \cap B_{i}^{\prime}, \sigma$ cannot enter $\operatorname{int}\left(B_{i} \cup B_{i}^{\prime}\right)$, so lies in $F$. If $F$ is an $(n-1)$-face to which $\sigma$ is tangent at $p$, then by convexity of $B_{i}$ and $B_{i}^{\prime}, \sigma$ could only leave $F$ by striking an adjacent 
$(n-2)$-face, which as we have just seen is impossible. If $F$ is an $(n-1)$-face to which $\sigma$ is transverse at $p$, then $\sigma$ enters $\operatorname{int}(M \cap \bar{M})$.

Now we are ready to prove the Bonnet-Myers comparison result Lemma 5.5 .

Proof of Lemma 5.5. Suppose to the contrary that there is a point of $\bar{M}$ whose distance to $\Gamma$ is greater than $\pi$. Then there is a geodesic $\gamma:[0, \ell] \rightarrow \operatorname{int}(\bar{M})$ of length

$$
\ell=d(\gamma(0), \gamma(\ell))>\pi \text {. }
$$

We show this is impossible by constructing a length-shortening variation of $\gamma$.

By shortening $\gamma$ slightly if necessary, we may ensure that if $\gamma$ has an endpoint in an $(n-2)$ - face, then a nontrivial end subsegment of $\gamma$ lies in that face. Thus by Lemma 5.5, either

(1) each clipping segment of $\gamma$ lies in an $n$-face except possibly for endpoints at which it strikes an $(n-1)$-face transversely, or

(2) $\gamma$ additionally contains clipping segments that lie in lower-dimensional faces.

In case (i), we are going to show that the standard treatment of Bonnet-Myers comparison via second variation works with an adjustment. In case (ii), we make a local modification of $\bar{M}$ in a neighborhood of $\gamma$ in order to recover case (i).

Case (i). We use [27] as our reference for Bonnet-Myers comparison in Riemannian manifolds. As there, let $\mathbf{x}:[0, \ell] \times[-\delta, \delta] \rightarrow \bar{M}$ be a variation of $\gamma$ with fixed endpoints, which is smooth on each $\left[u_{i-1}, u_{i}\right] \times[-\delta, \delta]$ for some partition of $[0, \ell]$. This is possible by taking the partition breaks $u_{i}$ at the transverse crossings between adjacent $n$-faces, so that $\mathbf{x}\left(u_{i}, v\right)$ lies on an $(n-1)$-face $F$. On each $\left[u_{i-1}, u_{i}\right] \times[-\delta, \delta]$, write $\mathbf{x}_{v}=V$ and $\mathbf{x}_{v v}=A$, where $\mathbf{x}_{v v}=\nabla_{V} V$ denotes the Euclidean or $M$ covariant derivative alternately, depending on the $n$-face. The second variation of length $L^{\prime \prime}(0)$ is calculated by adding the corresponding piecewise contributions at $v=0$; see [27. Theorem 4, p. 266 \& Comment (1)]. In the Riemannian case, a term cancels, namely

$$
\left\langle\gamma^{\prime}, A\right\rangle\left(u_{i}^{-}\right)-\left\langle\gamma^{\prime}, A\right\rangle\left(u_{i}^{+}\right)=0,
$$

because $\left\langle\gamma^{\prime}, A\right\rangle$ is continuous along $\gamma$. In our case, the tangent cone at $\gamma\left(u_{i}\right)$ is isometric to $\mathbf{R}^{n}$, and under that isometry $\gamma^{\prime}\left(u_{i}^{-}\right)=\gamma^{\prime}\left(u_{i}^{+}\right)$. If $\gamma^{\prime}$ is replaced in (5.1) by its projection tangent to $F$, equality continues to hold since the tangential projection of $A$ is determined only by $F$, being its intrinsic covariant derivative $\widetilde{\nabla}_{V} V$. However, the projection of $A$ on a unit vector normal to $F$ depends on the side, $u_{i}^{-}$or $u_{i}^{+}$, being $I I(V, V)$ for the second fundamental form of $F$ relative to the corresponding $n$-face. If $\lambda$ is the value of $I I(V, V)$ relative to the compact cap $n$-face with the inward normal, then $\lambda \geq 0$ by convexity. The value of $I I(V, V)$ relative to the opposite face with its inward normal is $\lambda \cos \theta$ where $\theta$ is the angle between the two normals. Since $\lambda+\lambda \cos \theta \geq 0$, if $\gamma^{\prime}$ is replaced in (5.1) by its projection orthogonal to $F$, then the lefthand side becomes nonpositive. Thus in our case, (5.1) is replaced by

$$
\left\langle\gamma^{\prime}, A\right\rangle\left(u_{i}^{-}\right)-\left\langle\gamma^{\prime}, A\right\rangle\left(u_{i}^{+}\right) \leq 0
$$


When (5.2) replaces (5.1), the derivation of the second variation formula in [27, Corollary 8, p. 270] yields an inequality in place of the equation given there:

$$
L^{\prime \prime}(0) \leq-\int_{0}^{\ell}\left\langle\left(V^{\perp}\right)^{\prime \prime}-R\left(V^{\perp}, \gamma^{\prime}\right) \gamma^{\prime}, V^{\perp}\right\rangle d u-\Sigma\left\langle\left(V^{\perp}\right)^{\prime}\left(u_{i}^{+}\right)-\left(V^{\perp}\right)^{\prime}\left(u_{i}^{-}\right), V\left(u_{i}\right)\right\rangle,
$$

where we fix $v=0$, and $\perp$ indicates the component orthogonal to $\gamma^{\prime}$.

Now choose $\mathbf{x}$ with $\mathbf{x}(0, v)=\gamma(0), \mathbf{x}(\ell, v)=\gamma(\ell)$, and $V(u, 0)^{\perp}=\sin \frac{\pi u}{\ell} E(u)$, where $E(u)$ is a unit vector field that is orthogonal to $\gamma$ and parallel along each unbroken parameter interval. By (5.3),

$$
\begin{aligned}
L^{\prime \prime}(0) & \leq-\int_{0}^{\ell}\left(-\frac{\pi^{2}}{\ell^{2}} \sin ^{2} \frac{\pi u}{\ell}+\sin ^{2} \frac{\pi u}{\ell} K\left(\gamma^{\prime}, V^{\perp}\right)\right) d u \\
& \leq \int_{0}^{\ell} \sin ^{2} \frac{\pi u}{\ell}\left(\frac{\pi^{2}}{\ell^{2}}-1\right) d u<0,
\end{aligned}
$$

since the sectional curvature of the $n$-faces is $\geq 1$ and $\pi<\ell$.

Since $L^{\prime}(0)=0$ by first variation $\left(\left[27\right.\right.$, p. 264]) and we have just shown $L^{\prime \prime}(0)<0$, there is a variation with fixed endpoints of $\gamma$ in $\bar{M}$ that reduces length.

Case (ii). For a clipping segment of $\gamma$ that lies in a lower-dimensional face, the switchpoints to its two adjacent segments in $\operatorname{int}(M \cap \bar{M})$ need not vary smoothly under a variation of $\gamma$, although they clearly vary upper semicontinuously, and so the preceding argument does not apply. We avoid this difficulty by making a local modification of $\bar{M}$ in a neighborhood of each of the (finitely many) clipping segments $\sigma$ that lie in lower-dimensional faces.

Such a $\sigma$ lies in $M \cap \bar{M}$ and joins two nontrivial geodesic segments $\tau_{1}$ and $\tau_{2}$ that lie in $\operatorname{int}(M \cap \bar{M})$. Thus if we replace a neighborhood $\bar{U}_{\sigma}$ of $\sigma$ in $\bar{M}$ by a neighborhood $U_{\sigma}$ of $\sigma$ in $M$, then $U_{\sigma}$ joins smoothly with neighborhoods in $\bar{M}$ of $\tau_{1}$ and $\tau_{2}$.

Since the second fundamental form of $M$ has nullity at most 1, it is nonvanishing, so $M$ and $\bar{M}$ are one-sidedly locally convex; and since $\bar{M}$ was obtained from $M$ by a clipping procedure, $\bar{U}_{\sigma}$ lies to the inward side of $M$. Moreover, $\bar{U}_{\sigma}$ may be taken to lie on a convex hypersurface. To see this, let us transform from $\mathbf{S}^{n+1}$ back to $\mathbf{R}^{n+1}$ for simplicity of terminology. Since $\sigma$ is a line segment in the nonnegatively curved hypersurface $M$, then by the Gauss Equation, $\sigma$ is tangent to the nullity subspace. Equivalently, the tangent hyperplane to $M$ is constant, say $H_{\sigma}$, along $\sigma$. Then $U_{\sigma}$ lies on the boundary of a body $K$ constructed as follows. Cover $\sigma$ by a finite union of convex neighborhoods in $M$, each lying on the boundary of a convex body, and let $B$ be the intersection of the corresponding union of convex bodies with a closed halfspace $\widetilde{H}^{+}$containing $H_{\sigma}$, where $\widetilde{H}$ is parallel to $H_{\sigma}$. By the nullity hypothesis, the slices of $M$ by hyperplanes orthogonal to $\sigma$ are strictly positively curved at points of $\sigma$. Moreover, the clipping segment $\sigma$ is not extendible as a line segment in $M$, since if it were it would remain in its clipping hyperplane(s), $H_{i}$ and/or $H_{i}^{\prime}$, and hence be extendible as a clipping segment. Therefore $U_{\sigma} \cap \operatorname{int} \widetilde{H}^{+}=\partial K \cap \operatorname{int} \widetilde{H}^{+}$if $\widetilde{H}$ is chosen sufficiently close to $H_{\sigma}$. Thus the body $K$ has a locally convex boundary, 
so is convex. By the clipping procedure, if $\bar{U}_{\sigma}$ is sufficiently small, it lies on the boundary of the intersection of $K$ with either one or two halfspaces. Therefore $\bar{U}_{\sigma}$ lies on a convex hypersurface in the inward side of $U_{\sigma}$.

By a classical theorem of Busemann-Feller [10], projection to a convex hypersurface from the outside does not increase length. It follows that if we choose $U_{\sigma}$ sufficiently small, $U_{\sigma}$ has a well-defined and length-nonincreasing projection map to $\bar{U}_{\sigma}$.

Let $\widetilde{M}$ be the hypersurface obtained by replacing $\bar{U}_{\sigma}$ by $U_{\sigma}$ for each clipping segment $\sigma$ that lies in a lower-dimensional face. As in case (i), there is a length-reducing variation with fixed endpoints of $\gamma$ in $\widetilde{M}$. Since there is a length-nonincreasing map from $\widetilde{M}$ to $\bar{M}$, there is a shorter curve than $\gamma$ in $\bar{M}$ joining the endpoints of $\gamma$. This is the desired contradiction.

\section{Comments and Questions}

6.1. In light of the results we have obtained thus far, one outstanding question which naturally presents itself is:

Question 6.1. What is the biggest integer $d$ such that every smooth compact submanifold of codimension $d$ in $\mathbf{R}^{n}$ bounds only finitely many topological types of positively curved fillings?

By Theorems 1.6 and 1.5, $2 \leq d \leq 15$, although it is possible that $d=2$. Indeed, if the question [41, Problem 25] of whether any 3-manifold with almost constant positive sectional curvature isometrically embeds in $\mathbf{R}^{6}$, had an affirmative answer, then one might be able to construct the examples of Theorem 1.5 in $\mathbf{R}^{6}$. Specifically, it would be enough to show that there exists an infinite subfamily of lens spaces which may be embedded with positive curvature in $\mathbf{R}^{6}$ so that they all coincide over an open neighborhood. This may be plausible since by a well-known theorem of Hirsch [23, every orientable 3-manifold embeds in $\mathbf{R}^{5}$; further, Zeeman [42] has shown that an infinite subfamily of punctured lens spaces even embed in $\mathbf{R}^{4}$.

6.2. It is also natural to want to estimate the number of topological types of positively curved fillings for a given submanifold. To this end we should point out:

Remark 6.2. In Theorems 1.2, 1.4, 1.6, and 1.7, the bound to the number of topological types of fillings may be estimated explicitly. It depends on the lower curvature bound of the ambient manifold, the norm of the second fundamental form of the given submanifold, as well as its intrinsic diameter and lower volume bound (and in 1.3, on the integral of curvature), but on no other characteristic of the submanifold.

Further, we should mention that, as it was proved in [1], if $\Gamma \subset \mathbf{R}^{n}$ is a compact submanifold of codimension 2 which lies on the boundary of a convex body, then it bounds at most two topologically distinct locally strictly convex compact hypersurfaces. Incidentally, no further regularity is required of $\Gamma$ in this case. 
6.3. Finally, in light of the Soul theorem of Cheeger-Gromoll-Meyer 19, 12, it is interesting to ask to what extent Theorem 1.7 might reflect intrinsic finiteness phenomena. Recall that a complete, open, positively curved Riemannian manifold $M$ without boundary is diffeomorphic to $\mathbf{R}^{n}$. Perelman proved the same statement if $M$ is merely nonnegatively curved with a point of strictly positive curvature [29]. Moreover, there are only finitely many homeomorphism types of complete, open, nonnegatively curved Riemannian manifolds of fixed dimension, with a uniform upper bound on the diameter of the soul, and a uniform lower bound on the volume of some ball of uniform radius lying at uniformly bounded distance from the soul (see [7]). However, except for dimension 2, "intrinsic" finiteness does not hold for the class of complete open manifolds which are positively curved off compact sets with uniform upper diameter and lower volume bounds. This remains true even in the context of submanifold geometry:

Example 6.3. Modify the example of Theorem 1.5 by ambiently attaching a warped product $\mathbf{S}^{2} \times_{\phi}[0, \infty)$ to $\mathbf{S}^{2}(1) \subset \mathbf{R}^{18}$. One can choose $\phi$ so that this results in a sequence of complete 3-manifolds embedded in $\mathbf{R}^{18}$ with one end (topologically just punctured lens spaces), and the curvature can be made positive off a ball of fixed radius. Moreover, the curvature of the resulting manifolds is bounded from below, since the second fundamental form of the fixed boundary $\mathbf{S}^{2}(1) \subset \mathbf{R}^{18}$ of the filling manifolds $\left(\mathbf{S}^{3} / \mathbf{Z}_{i}\right) \backslash B^{3}$ is uniformly bounded in norm.

\section{ACKNOWLEDGMEnT}

We thank Bo Guan and Joel Spruck who first called our attention to the question of finiteness of topological types for positively curved hypersurfaces filling a prescribed boundary. Thanks also to Igor Belegradek for useful conversations.

\section{REFERENCES}

[1] S. Alexander and M. Ghomi. The convex hull property and topology of hypersurfaces with nonnegative curvature. Adv. Math., 180(1):324-354, 2003.

[2] S. Alexander and M. Ghomi. The convex hull property of noncompact hypersurfaces with positive curvature. Amer. J. Math., 126(4):891-897, 2004.

[3] S. Alexander, V. Kapovitch, and A. Petrunin. An optimal lower curvature bound for convex hypersurfaces in Riemannian manifolds. Illinois J. Math., to appear.

[4] S. B. Alexander and R. J. Currier. Non-negatively curved ends of Euclidean hypersurfaces. Geom. Dedicata, 40(1):29-43, 1991.

[5] A. D. Alexandrov and Y. G. Reshetnyak. General theory of irregular curves, volume 29 of Mathematics and its Applications (Soviet Series). Kluwer Academic Publishers Group, Dordrecht, 1989. Translated from the Russian by L. Ya. Yuzina.

[6] A. D. Alexandrov and V. A. Zalgaller. Intrinsic geometry of surfaces. Translated from the Russian by J. M. Danskin. Translations of Mathematical Monographs, Vol. 15. American Mathematical Society, Providence, R.I., 1967.

[7] I. Belegradek and V. Kapovitch. Finiteness theorems for nonnegatively curved vector bundles. Duke Math. J., 108(1):109-134, 2001.

[8] D. Burago, Y. Burago, and S. Ivanov. A course in metric geometry, volume 33 of Graduate Studies in Mathematics. American Mathematical Society, Providence, RI, 2001. 
[9] Y. D. Burago and S. Z. Shefel. The geometry of surfaces in Euclidean spaces [ MR1039818 (91d:53004)]. In Geometry, III, volume 48 of Encyclopaedia Math. Sci., pages 1-85, 251-256. Springer, Berlin, 1992.

[10] H. Busemann and W. Feller. Krümmungseigenschaften Konvexer Flächen. Acta Math., 66(1):147, 1936.

[11] M. Cai. Ends of Riemannian manifolds with nonnegative Ricci curvature outside a compact set. Bull. Amer. Math. Soc. (N.S.), 24(2):371-377, 1991.

[12] J. Cheeger and D. Gromoll. On the structure of complete manifolds of nonnegative curvature. Ann. of Math. (2), 96:413-443, 1972.

[13] M. P. do Carmo and F. W. Warner. Rigidity and convexity of hypersurfaces in spheres. J. Differential Geometry, 4:133-144, 1970.

[14] M. Ghomi. Strictly convex submanifolds and hypersurfaces of positive curvature. J. Differential Geom., 57(2):239-271, 2001.

[15] M. Ghomi. Gauss map, topology, and convexity of hypersurfaces with nonvanishing curvature. Topology, 41(1):107-117, 2002.

[16] M. Ghomi and R. Greene. Relative isometric embeddings of Riemannian manifolds. To appear in Trans. Amer. Math. Soc.

[17] M. Ghomi and M. Kossowski. $h$-principles for hypersurfaces with prescribed principal curvatures and directions. Trans. Amer. Math. Soc., 358(10):4379-4393 (electronic), 2006.

[18] H. Gluck and L.-H. Pan. Embedding and knotting of positive curvature surfaces in 3-space. Topology, 37(4):851-873, 1998.

[19] D. Gromoll and W. Meyer. On complete open manifolds of positive curvature. Ann. of Math. (2), 90:75-90, 1969.

[20] B. Guan and J. Spruck. Locally convex hypersurfaces of constant curvature with boundary. Comm. Pure Appl. Math., 57(10):1311-1331, 2004.

[21] P. Hartman and L. Nirenberg. On spherical image maps whose Jacobians do not change sign. Amer. J. Math., 81:901-920, 1959.

[22] L. Hauswirth. Bridge principle for constant and positive Gauss curvature surfaces. Comm. Anal. Geom., 7(3):497-550, 1999.

[23] M. W. Hirsch. The imbedding of bounding manifolds in euclidean space. Ann. of Math. (2), 74:494-497, 1961.

[24] V. Kapovitch. Perelman's stability theorem. to appear in Surveys in Comparison Geometry.

[25] N. N. Kosovskiǔ. Gluing of Riemannian manifolds of curvature $\geq \kappa$. Algebra i Analiz, 14(3):140$157,2002$.

[26] J. McCuan. Positively curved surfaces with no tangent support plane. Proc. Amer. Math. Soc., 133(1):263-273 (electronic), 2005.

[27] B. O'Neill. Semi-Riemannian geometry, volume 103 of Pure and Applied Mathematics. Academic Press Inc. [Harcourt Brace Jovanovich Publishers], New York, 1983. With applications to relativity.

[28] G. Perelman. Alexandrov's spaces with curvature bounded from below II. preprint (1991).

[29] G. Perelman. Proof of the soul conjecture of Cheeger and Gromoll. J. Differential Geom., 40(1):209-212, 1994.

[30] G. Perelman. Construction of manifolds of positive Ricci curvature with big volume and large Betti numbers. In Comparison geometry (Berkeley, CA, 1993-94), volume 30 of Math. Sci. Res. Inst. Publ., pages 157-163. Cambridge Univ. Press, Cambridge, 1997.

[31] H. Rosenberg. Hypersurfaces of constant curvature in space forms. Bull. Sci. Math., 117(2):211239, 1993.

[32] R. Sacksteder. On hypersurfaces with no negative sectional curvatures. Amer. J. Math., 82:609$630,1960$.

[33] K. Shiohama, T. Shioya, and M. Tanaka. The geometry of total curvature on complete open surfaces, volume 159 of Cambridge Tracts in Mathematics. Cambridge University Press, Cambridge, 2003. 
[34] M. Spivak. A comprehensive introduction to differential geometry. Vol. IV. Publish or Perish Inc., Wilmington, Del., second edition, 1979.

[35] N. S. Trudinger and X.-J. Wang. On locally convex hypersurfaces with boundary. J. Reine Angew. Math., 551:11-32, 2002.

[36] V. V. Usov. The three-dimensional swerve of curves on convex surfaces. Sibirsk. Mat. Ž., 17(6):1427-1430, 1440, 1976.

[37] J. Van Heijenoort. On locally convex manifolds. Comm. Pure Appl. Math., 5:223-242, 1952.

[38] H.-H. Wang. Boundary convexity on manifolds with nonnegative Ricci curvature. PhD thesis, Indiana University, 1997.

[39] J. Wong. Collapsing manifolds with boundary. PhD thesis, University of Illinois at UrbanaChampaign, 2006.

[40] J. Wong. An extension procedure for manifolds with boundary. Pacific J. Math., 235:173-199, 2008.

[41] S.-T. Yau. Open problems in geometry. In Differential geometry: partial differential equations on manifolds (Los Angeles, CA, 1990), volume 54 of Proc. Sympos. Pure Math., pages 1-28. Amer. Math. Soc., Providence, RI, 1993.

[42] E. C. Zeeman. Twisting spun knots. Trans. Amer. Math. Soc., 115:471-495, 1965.

Department of Mathematics, University of Illinois at Urbana-Champaign, IL 61801

E-mail address: sba@math.uiuc.edu

$U R L:$ www.math.uiuc.edu/ ${ }^{\sim} \mathrm{sba}$

School of Mathematics, Georgia Institute of Technology, Atlanta, GA 30332

E-mail address: ghomi@math.gatech.edu

$U R L$ : www . math.gatech.edu/ ghomi

Department of Mathematics, University of Toronto, Toronto, ON, M5S 2E4

E-mail address: jawong1@math.toronto.edu

$U R L$ : www . math.toronto. edu/ jawong1 